\title{
Cannabinoid glycosides: In vitro production of a new class of cannabinoids with improved physicochemical properties
}

Janee' M. Hardman, Robert T. Brooke, Brandon J. Zipp*.

Vitality Biopharma Inc., 1901 Avenue of the Stars, 2nd Floor, Los Angeles, California 90067

* Corresponding author: zipp@vitality.bio

\section{Abstract:}

The cannabinoid signaling system has recently garnered attention as a therapeutic target for numerous indications, and cannabinoids are now being pursued as new treatment options in diverse medical fields such as neurology, gastroenterology, pain management, and oncology. Cannabinoids are extremely hydrophobic and relatively unstable compounds, and as a result, formulation and delivery options are severely limited. Enzymatic glycosylation is a strategy to alter the physicochemical properties of small molecules, often improving their stability and aqueous solubility, as well as enabling site-specific drug targeting strategies. To determine if cannabinoids are a candidate for glycosylation, a library of glucosyltransferase (UGT) enzymes was screened for glycosylation activity towards various cannabinoids. The UGT76G1 enzyme from Stevia rebaudiana has been identified as having glucosyltransferase activity towards a broad range of cannabinoids. Compounds that were successfully glycosylated by UGT76G1 include the phytocannabinoids cannabidiol (CBD), $\Delta^{9}$-tetrahydrocannabinol ( $\left.\Delta^{9}-T H C\right)$, cannabidivarin (CBDV), and cannabinol (CBN), and the human endocannabinoids anandamide (AEA), 2-arachidonoyl-glycerol (2AG), 1-arachidonoyl-glycerol (1AG), and synaptamide (DHEA). Interestingly, UGT76G1 is able to transfer primary, secondary, and tertiary glycosylations at each acceptor of most of the cannabinoids tested. Additionally, Os03g0702000p, a glycosyltransferase from Oryza sativa, was able to transfer secondary glucose residues onto cannabinoid monoglycosides previously established by UGT76G1. This new class of cannabinoid-glycosides has been termed cannabosides. The compounds have greatly improved solubility in aqueous solutions. This increased aqueous solubility may enable new oral pharmaceutical delivery options for cannabinoids, as well as targeted delivery and release of cannabinoids within the intestines through glycoside prodrug metabolism. 


\section{Introduction:}

The human endocannabinoid system (ECS) is a broad-spectrum modulator that plays a role in brain plasticity, nociception, inflammation, regulation of stress and emotions, and addiction (Aizpurua-Olaizola, 2017, Hryciw 2016, Gye 2005, Slavic 2013). It includes the endogenous cannabinoids (endocannabinoids), their respective cannabinoid receptors, and the associated enzymes required to synthesize and break down the endocannabinoids (Mackie, 2008). The ECS is a diverse system whose main receptors, CB1 and CB2, are found throughout the body including the central and peripheral nervous systems, the immune system, and gastrointestinal tract (as reviewed recently by Hasenoehrl 2016 and DiPatrizio 2016), the testes, and the heart. Phytocannabinoids from Cannabis sativa are capable of modulating ECS receptors and have a long history of medicinal and recreational use (Touw 1981). $\Delta^{9}$-Tetrahydrocannabinol ( $\left.\Delta^{9}-\mathrm{THC}\right)$, perhaps the best known psychoactive phytocannabinoid, is only one of over 70 cannabinoids produced by Cannabis sativa (Atakan 2012, Welling 2016). Cannabidiol (CBD), a non-psychoactive phytocannabinoid, has recently garnered attention as a potential anti-epileptic, anti-psychotic, and neuroprotectant (Porter 2013, Leo 2016, Zuardi 2012, luvone 2009, for review Mechoulam 2002).

Despite their pharmacological promise, cannabinoids are extremely hydrophobic and have very poor solubility in aqueous solutions, necessitating the use of oils and solvents as a carriers in drug formulations, which are not tolerated well and may contribute to oral lesions at high dose or in long-term use (Scully 2007). Numerous efforts have been undertaken to improve the aqueous solubility of CBD and THC. Cyclized maltodextrins have been found to improve the solubility of cannabinoids non-covalently, and covalent modifications have produced chemically derivatized CBD prodrugs with improved solubility (Jarho 1998, WO2009018389, WO2012011112).

Fluorine substitutions of CBD have also been created through synthetic chemical manipulations in an effort to functionalize CBD (WO2014108899). Others have gone so far as to modify THC with carboxamido, imidazole, pyrazole, triazole and morpholine pentyl side chain analogs, in addition to converting the phenolic hydroxyl group to substituted esters in an effort to increase solubility (Martin 2006). These strategies have improved the solubility of cannabinoids with limited success, and their unnatural compositions release synthetic prodrug moieties upon hydrolysis. 
As with synthetic chemistry, in vivo detoxification strategies serve as another model for improving the solubility of cannabinoids. Screening of human liver UGTs against cannabinoids found that cannabinol (CBN) is efficiently glucuronidated by human UGT1A10 in vitro, and minor activity was demonstrated towards CBD with UGT1A9 and UGT2B7 (US8,410,064). Screening of plant cell cultures has shown that $\mathrm{CBN}$ is glycosylated when incubated with Pinellia ternata cell cultures (Tanaka 1993). Similarly, CBD was shown to be glycosylated when incubated with tissue cultures from Pinellia ternata and Datura inoxia, yielding CBD-6'-O- $\beta$-D-glucopyranoside and CBD-(2',6')-O- $\beta$-D-diglucopyranoside (Tanaka 1996). These cell culture biotransformation studies demonstrated the potential for limited glycosylation of cannabinoids, but previously, no specific enzymes or methods had been identified that could enable the production of high-purity pharmaceutical preparations or a diverse class of cannabinoid-glycosides.

The plant Stevia rebaudiana produces a diverse family of steviol glycosides and possesses an untapped pool of UGT enzymes, making Stevia an ideal candidate for small-molecule glycosylation screening. UGT76G1 is a glucosyltransferase from Stevia capable of transferring a secondary glucose to the C3-hydroxyl of the primary glycosylation on both $\mathrm{C} 13-\mathrm{OH}$ and $\mathrm{C} 19-\mathrm{COOH}$ positions of the steviol glycoside, and thus its substrates include steviolmonoside, stevioside, rubusoside, RebA, RebD, RebG, and RebE (Richman et al. 2005, Stevia First Corp unpublished data).

To address the issue of poor cannabinoid solubility and identify methods of producing novel cannabinoid pharmaceutical prodrugs, we screened glucosyltransferase enzymes from Stevia and other organisms to identify candidates for the glycosylation of cannabinoids. We also set out to characterize any resulting cannabinoid glycosides for improved physicochemical properties.

\section{Results:}

\section{UGT76G1 Glycosylation of Cannabidiol}

To identify enzymes with glycosylation activity towards cannabinoids, a Stevia rebaudiana UGT enzyme library was reacted with CBD (Supplemental figure 1). Upon incubation of CBD with the Stevia rebaudiana glucosyltransferase UGT76G1, depletion of the input CBD was observed by RP-HPLC (dotted line, CBD retention time 13.6 min, Figure 1A). CBD was positively identified in 
the HPLC line trace based on comparison with the retention time of a purchased CBD standard (Cayman Chemical), along with a doublet fingerprint peak at the cannabinoid absorbance maximum of $275 \mathrm{~nm}$. Prolonged incubation of CBD with UGT76G1 yielded 4 distinct glycoside product mobility groups with retention times of $8.75,9.0,10.3$, and 10.7 minutes, respectively, with other minor products present (Figure 1A). Glycosylation of CBD caused a slight shifting of the fingerprint doublet absorbance maximum at $275 \mathrm{~nm}$ to $270 \mathrm{~nm}$, which is consistent with other aglycone-to-glycoside conversions (Figure 1B). CBD glycosylation was found to be UDPG-dependent, and no direct glycosylation of CBD was observed with other tested enzymes. In the presence of excess UDPG, UGT76G1 depleted $>95 \%$ of the input CBD and had an equilibrium constant (Keq) of $\sim 24$. LC-ESI-MS was performed on the CBD glycoside mixture resulting from the UGT76G1 CBD reaction, and $[\mathrm{M}+\mathrm{H}] \mathrm{m} / \mathrm{z}$ peaks were detected for the CBD aglycone along with the CBD monoside, diglycoside, triglycoside and tetraglycoside $(\mathrm{m} / \mathrm{z}=315$, 477, 639, 801, and 982, respectively, Supplemental Figure 2A). ${ }^{1} \mathrm{H}-\mathrm{NMR}$ of the purified compounds with HPLC retention times of 9.0 and 10.7 minutes confirmed the production of the CBD diglycosides VB104 and VB110. VB104 is a CBD diglycoside where one available hydroxyl acceptor group has been conjugated with two glucose residues, and its production was confirmed by the presence of two distinct peaks in the aromatic region representing protons 3' and 5 ' from the substituted resorcinol ring of CBD (6.22 and $6.40 \mathrm{ppm}, 1 \mathrm{H}$ each, Supplemental Figures 2B, 2C). VB110 production was confirmed by the presence of one doublet peak in the aromatic region (6.68 ppm, $2 \mathrm{H}$ ) representing protons 3' and 5', indicating that both the 2' and 6' hydroxyl acceptor groups had been conjugated with a glucose residue (Supplemental Figure 2D). The determined CBD glycoside structures of UGT76G1 are depicted in Figure 1D.

\section{UGT76G1 Glycosylation of Additional Phytocannabinoids}

Based on the efficient glycosylation of the hydroxyl groups of CBD by UGT76G1, additional phytocannabinoids were screened as substrates for UGT76G1, including CBDV, CBN, and $\Delta^{9}$-THC. CBDV, a cannabinoid differing from CBD by a three-carbon rather than a five-carbon hydrocarbon tail, was selected to be screened next due to its structural similarity to CBD. CBDV was incubated with UGT76G1 and UDPG in a similar manner as CBD. The reaction was monitored by RP-HPLC and CBDV depletion was observed (CDBV retention time $12.7 \mathrm{~min}$ ), in addition to the appearance of three additional mobility peaks at 8.5, 9.7, and 10.0 minutes (Figure 3A). Formation of these products was dependent on addition of both UGT76G1 and UDPG. The three new products formed displayed the same absorbance characteristics as 
CBDV and were determined to be the primary glycosides CBDV-2'-O-glucopyranoside or CBDV-6'-O-glucopyranoside, the secondary glycosides CBDV-2'-O-(3-1)-diglucopyranoside or CBDV-6'-O-(3-1)-diglucopyranoside, and CBDV-2',6'-O-diglucopyranoside (compounds VB202/VB206, VB204/VB208, and VB210 respectively). With additional reaction time it was determined that minor product peaks containing higher-order glycosides were also formed, analogous to what was seen when CBD was the substrate. The reaction proceeded to $>95 \%$ substrate conversion with $\mathrm{K}_{\mathrm{eq}} \sim 24$.

Both the 2' and 6' hydroxyl groups of CBD and CBDV were successfully glycosylated and both compounds exhibit free rotation about the $\mathrm{C} 1$ ' bond between the resorcinol ring and the terpene ring. To determine if the cannabinoids were binding in the active site of UGT76G1 in a rigid conformation or if there was rotation along the $\mathrm{C} 1^{\prime}$, bond, $\Delta^{9}$-tetrahydrocannabinol $\left(\Delta^{9}-\mathrm{THC}\right)$ was screened as a potential substrate. $\Delta^{9}-\mathrm{THC}$ was hypothesized to provide insight into active site binding directionality because it has undergone a ring closure between the 6' hydroxyl group and the terpene ring, thus eliminating any rotation about the $\mathrm{C} 1^{\prime}$ ' bond. Additionally, $\Delta^{9}-\mathrm{THC}$ only has the 2' hydroxyl group free for glycosylation (1-OH based on formal dibenzopyran numbering for $\Delta^{9}$-THC). Upon incubation of $\Delta^{9}-$ THC with UGT76G1 and UDPG, RP-HPLC showed depletion of the input $\Delta^{9}-\mathrm{THC}$ (retention time $14.5 \mathrm{~min}$ ) and the formation of three main product peaks at 10.6, 10.9, and 11.7 minutes (dotted line, Figure 2B). The three new products formed displayed a similar UV-VIS absorbance spectrum to $\Delta^{9}$-THC (data not shown). LC-ESI-MS analysis of the glycoside mixture confirmed the identity of the glycosides as $\triangle 9$-THC monoside, diglycoside and triglycoside $([\mathrm{M}+\mathrm{H}] \mathrm{m} / \mathrm{z}=315,477,639$ and 801, respectively, Supplemental Figure 2D). Structures for $\triangle 9-\mathrm{THC}$ are as depicted in Figure 3D.

The final phytocannabinoid that was screened as a substrate for UGT76G1 was the $\Delta^{9}$-THC natural degradation product, cannabinol (CBN), which resembles $\Delta^{9}-\mathrm{THC}$ but the upper terpene ring has been fully oxidized to its aromatic form. Upon incubation of CBN with UGT76G1 and UDPG, RP-HPLC showed depletion of the input CBN (CBN retention time $14.2 \mathrm{~min}$ ) and formation of three main product peaks at 10.8, 11.5 and 12.2 minutes (Figure 3C). The three new products displayed the same absorbance characteristics as CBN (data not shown).

\section{Secondary Glycosylation With Os03g0702000p}


Branched chain glycosides are often glycosylated by specific enzymes in a stepwise manner, and certain UGTs only recognize a glycoside as their substrate. As UGT76G1 was found to establish primary, secondary, and tertiary glycosylations on the hydroxyl groups of CBD, additional UGTs were screened for their ability to recognize cannabinoid glycosides as substrates for further glycosylation. Os03g0702000p (formerly referred to as EUGT11) is a UGT from Oryza sativa that has secondary glycosylation activity towards steviol glycosides (WO 2013022989). It was tested and determined that Os03g0702000p is capable of transferring an additional glucose moiety from UDPG onto the C2-hydroxyl of the primary sugar established by UGT76G1 ( $\beta-2 \rightarrow 1$ connectivity, Figure $2 \mathrm{C}$ ), similar to the secondary glycosylation activity that UGT76G1 has towards the C3-hydroxyl of the primary glucose residue glycosylation $(\beta-3 \rightarrow 1$ connectivity). Upon incubation of a CBD glycoside mixture generated by UGT76G1 with Os03g0702000p, four additional glycoside products were formed with HPLC retention times of $9.9,10.2,10.3$, and 10.6 minutes, which retained the cannabinoid absorbance maxima at 270 $\mathrm{nm}$. This glycosylation activity is consistent with the activity of UGT Os03g0702000p towards steviol glycosides in establishing C2-hydroxyl secondary glycosylations ( $\beta-2 \rightarrow 1$ connectivity) on existing primary glucose residues. Similar glycosylation activity was observed when CBDV, $\Delta^{9}-\mathrm{THC}$, and CBN glycoside mixtures were incubated with Os03g07000p and excess UDPG (data not shown). All glycoside products were identified based on the retained cannabinoid absorbance maxima at $275 \mathrm{~nm}$. No glycosylation activity was seen when Os03g0702000p alone was incubated with cannabinoids.

\section{UGT76G1 Glycosylation of Endocannabinoids}

As UGT76G1 has been determined to recognize a broad class of phytocannabinoids, it was hypothesized that the same enzyme active site may also accommodate and glycosylate endocannabinoids, the endogenous human signaling molecules recognized by the cannabinoid receptors $\mathrm{CB} 1$ and $\mathrm{CB} 2$ in humans. Accordingly, the endocannabinoids $A E A, 2 A G, 1 A G$, and DHEA were obtained and screened as possible substrates for glycosylation by UGT76G1. All endocannabinoid compounds showed UDPG-dependent depletion of the substrates and the formation of new product peaks by RP-HPLC (Figures 4A, 4B, and 4C, respectively). Upon incubation with UGT76G1 and UDPG, AEA (retention time of $13.9 \mathrm{~min}$ ) yielded one glycoside product peak with a retention time of $12.5 \mathrm{~min}$ (Figure 4A). Glycosylation of AEA occurred slowly, and reactions did not proceed to product/substrate ratios seen with phytocannabinoids. 2-AG and 1-AG were obtained in a 90:10 2-AG:1-AG ratio and each displayed an absorbance 
maximum at $233 \mathrm{~nm}$ and RP-HPLC retention times of 14.2 (1-AG) and 14.3 (2-AG) minutes. Upon incubation with UGT76G1 and UDPG, both compounds showed UDPG-dependent depletion by UGT76G1 (Figure 4B). Nine new arachidonoylglycerol-glycoside product peaks were observed on the HPLC trace at 11.4, 11.7, 11.8, 11.9, 12.0, 12.5, 12.9, 13.1, and 13.3 minutes, and all retained the signature $233 \mathrm{~nm}$ absorbance maxima (Figure 4B). Finally, DHEA, which has an RP-HPLC retention time of $13.7 \mathrm{~min}$ and an absorbance maximum of $237 \mathrm{~nm}$, was incubated with UGT76G1 and UDPG. Two new glycoside product peaks were observed in the HPLC line trace at 10.1 and 12.4 minutes, and were identified as glycosides of DHEA as both displayed the signature absorbance maximum of $237 \mathrm{~nm}$ (Figure 4C).

\section{Physicochemical properties of cannabinoid glycosides}

Phytocannabinoids are hydrophobic compounds, which limits their formulation and delivery options for pharmaceutical uses. The cannabinoid glycoside products in the present study all displayed advanced elution from the reverse-phase HPLC separation, suggesting that they are less hydrophobic than their precursors. Based on this observation, it was hypothesized that the cannabinoid glycoside products would have improved aqueous solubility. The standard quantification for chemical solubility is partitioning in water and octanol $\left(\log \left(\mathrm{c}_{\text {octanol }} / \mathrm{c}_{\text {water }}\right)\right.$, or $\mathrm{C} \log \mathrm{P})$, and recently software has been developed to estimate the ClogP values. Select cannabosides were analyzed and ClogP values were calculated (Table 1). The addition of glucose residues to the cannabinoid backbone significantly decreased the ClogP relative to the parent compounds. $\Delta^{9}$-THC had a ClogP value of 7.2 , but the addition of one glucose residue (VB302) decreased the CLogP value to 5.7, and the addition of two glucose residues (VB304) reduced the value even further to 4.7. To explore how the location of the added sugar residues also affects the CLogP value, CLogP values for CBD were calculated. Compared to $\Delta^{9}-\mathrm{THC}$, which has one hydroxyl acceptor group, CBD has two hydroxyl acceptor groups at C2' and C6'. The calculated CLogP value for CBD was 6.6, and the addition of one glucose residue reduced this value to 5.1. With addition of a diglycoside to the hydroxyl group C2' (VB104), the CLogP value decreased even further to 4.3. When one glucose residue was added to each of the C2' and C6' hydroxyl groups (VB110), the CLogP value decreased to 3.4. The ClogP values predicted in silico were comparable to values determined experimentally by RP-HPLC using compounds with known ClogP values as standards. Comparison of the predicted ClogP values for the cannabosides with their elution times revealed a linear relationship between the ClogP value and the advanced elution time (Figure 5A). The decreases in CLogP values observed for 
the cannabinoid glycosides indicate improved water solubility of cannabosides compared to the parent aglycones.

Because the ClogP values and $\mathrm{C} 18$ retention times indicated that cannabosides have decreased hydrophobicity, the aqueous solubility was directly tested (Figure 5B,C). Solids in vials were imaged after hydration and mixing by pipetting at $1 \%$ in water, followed by 1 minute of vortexing (Figures 5B, C, and D, respectively). The vials contained: 1. water (for reference), 2. CBD (negative solubility control), 3. VB104, 4. VB110, 5. VB304, 6, saponin (positive foaming control), 7. SDS (positive foaming control), and 8. sucrose (positive solubility, negative foaming control). Pure crystalline CBD was insoluble and water failed to solubilize it. Cannaboside VB104 was hydrated by the added water but the solutions remained cloudy and opaque; additionally, it possessed detersive properties (Figure 5D vial 3). VB110 was fully solubilized in water and created a translucent dark liquid. VB304 had partial solubility in water and displayed significant detersive properties. Saponins and SDS were both water soluble and detersive at $1 \%$ in water. Finally, sucrose was fully water soluble at $1 \%$ and did not display any foaming. The significant foaming shown by VB304 persisted for over 8 hours, whereas the SDS foaming subsided after 1 hour (data not shown).

\section{Discussion:}

In the present study, UGT76G1 was capable of transferring primary, secondary, and tertiary ( $\beta$ 3-1) glycosylations onto receptor molecules including phyto- and endocannabinoids. It was also found that UGT76G1 is able to specifically glycosylate both the C2' and C6' hydroxyl groups on CBD and CBDV, in addition to the C1 hydroxyl group of $\Delta^{9}-\mathrm{THC}$ and CBN. Os03g0702000p from Oryza sativa was capable of transferring secondary ( $\beta 2-1)$ glycosylations onto primary glucose residues established by UGT76G1. The cannabinoid-glycosides produced by these reactions display greatly improved solubility relative to the aglycone precursors and represent a new class of water-soluble cannabinoids termed cannabosides.

The reactions for production of cannabosides are dependent on UDPG, and the products maintain a cannabinoid absorbance spectrum. Multiple CBD-glycoside product peaks are seen on HPLC, and stepwise glycosylation and structural characterization have shown that the products consist of CBD mono, di-, tri-, and tetra-glycosides. All of the CBD glycosides show 
advanced elution from the $\mathrm{C} 18$ column relative to the CBD substrate, indicating a decrease in hydrophobicity. Calculation of the ClogP values for the predominant CBD-glycosides show greatly decreased hydrophobicity compared to the CBD aglycone.

The number of CBD-glycoside product mobility groups indicates multiple glycosylations that occur on both hydroxyl groups. To determine how cannabinoids are coordinated in the active site of UGT76G1, CBD was superpositioned over the bi-functional substrate for UGT76G1, rebaudioside E (RebE) (Supplemental Figure 3). The mechanism for dual hydroxyl glycosylation is likely one of two possibilities: either CBD is docking in the UGT76G1 active site both forwards and backwards, creating a cis-like sugar conformation for the glycosylations relative to the cannabinoid backbone (mechanism depicted in Supplemental Figure 4), or alternatively, the rotational freedom about the bond at $\mathrm{C} 1$ ' may allow the resorcinol-hydroxyl-glycoside group to rotate 180 degrees after glycosylation, placing the second free hydroxyl group in the active site for glycosylation that occurs in a trans-like conformation relative to the first glycosylation (C6 carbon as described by Mazur 2009, mechanism depicted in Supplemental Figure 5). The difference between these two mechanisms is slight, but they produce structurally distinct glycoside products that may have significantly different properties.

The majority of reactions with UGT76G1 and CBD yielded only diglycosides and higher. Tanaka observed CBD-glycosides after incubating CBD in root cell cultures of Pinellia ternata for periods up to 30 days and did not perform any short-term assays (Tanaka 1996). As such, Tanaka et al. described difficulty in identifying monoglycosylations of cannabidiol in their biotransformation reactions. Similarly, in this work, UGT76G1 did not produce single glycosylations of CBD in prolonged in vitro enzymatic reactions. This is likely due to the high affinity of CBD-monoglycosides towards the active site of UGT76G1. Short-term kinetic assays with UGT76G1 did produce CBD-monoglycosylations (VB102, VB103) when reactions were stopped using $80 \%$ acetonitrile (data not shown). CBD may require two glycosylations to achieve adequate aqueous solubility for escaping the active site of UGT76G1.

Performing enzyme kinetics with UGT76G1 and CBD was complicated by the unique biochemistry of UGT76G1. First, the cannabinoid glycoside products are also substrates for further glycosylation by UGT76G1; therefore, the reactions produce multiple competitive inhibitors very early during the reaction. It was also observed that excess CBD acted to 
substantially inhibit UGT76G1 reactions; therefore, the substrate concentration needed to be limited to prevent kinetic inhibition of the enzyme (data not shown). Based on this observation, a fed-batch conversion reaction in which CBD was dosed in small amounts on an hourly basis was performed to determine whether maintaining a low concentration of CBD was beneficial to the conversion reaction. Interestingly, this fed-batch reaction preferentially produced VB104, indicating that CBD is the preferred substrate for UGT76G1 when competing with VB104. It was also observed that pre-incubation of UGT76G1 with UDPG resulted in a burst phase of glycoside production that quickly leveled off and appeared to reach a linear, steady-state rate of production of VB104. This initial burst phase likely exhausted the pre-bound UDPG, after which the dissociation of UDP from the active site and concurrent binding of UDPG came to equilibrium.

UGT76G1 exhibited glycosylation activity towards THC that was similar to its activity towards CBD. The multiple THC-glycoside products showed advanced elution from the $\mathrm{C} 18$ column and maintained the cannabinoid absorbance spectrum. Similar to CBD, the primary products of UGT76G1 glycosylation were mostly THC-diglycosides and higher. Because LC-ESI-MS clearly revealed a THC-triglycoside, UGT76G1 is capable of transferring up to tertiary glycosides to the same recipient substrate. The fourth product peak was determined to be a glycoside, but the precise structure was not elucidated at the time this manuscript was prepared. It is unlikely to be a THC-tetraglycoside, as the LC-ESI-MS did not indicate a tetraglycoside molecular weight; rather, it may be a secondary glycosylation other than ( $\beta 3-1)$ attachment, or a degradation product such as $\mathrm{CBN}$-glycoside. Given that the rigid structure of $\Delta^{9}$-THC does not have the same rotational freedom as $\mathrm{CBD}$ around the $\mathrm{C} 1$ ' resorcinol ring attachment, the cannabinoid backbone is recognized in the active site of UGT76G1 with the $\Delta^{9}-\mathrm{THC}$ C1 hydroxyl group situated towards the UDPG sugar donor (pyran numbering, Figure 1B).

As originally hypothesized, cannabosides have greatly improved solubility in aqueous solutions. The solubility is dependent on the number of sugars attached, as well as the position and attachment site of the individual sugars. The highest solubility was observed for VB119 and VB110, CBD-glycosides with glucose molecules on opposing C2' and C6' hydroxyl groups. For VB110, it was found that solutions in excess of $50 \% \mathrm{w} / \mathrm{V}(0.78 \mathrm{M})$ were possible using only water as the solvent (data not shown). Factoring in the molecular mass addition resulting from the two attached sugars, the CBD content is $24.6 \%$ by mass, or $0.39 \mathrm{M}$. This empirically 
determined aqueous solubility is reinforced by ClogP values calculated in silico using ChemDraw Ultra for phytocannabinoids conjugated with a maximum of two glucose residues. The ClogP values also show that the number and position of the glycosylations impact the predicted solubility, and validate the aqueous solubility differences seen between the two CBD-diglycosides VB110 (ClogP = 3.4) and VB104 (ClogP = 4.3). Whereas VB110 has glycosylations on opposite sides of the molecule, VB104 is more amphipathic with a hydrophilic glycosylation opposed by the free hydroxyl group. This is more analogous to the THC-glycosides that have lost the free hydroxyl group to ring closure and exhibit a more severely amphipathic structure. During the preparation of aqueous solutions, it was observed that specific cannabosides displayed detersive properties as indicated by foaming while mixing. This detersive foaming is reminiscent of saponins, which are naturally occurring amphipathic plant glycosides known for their detersive properties, as well as foaming displayed by steviol glycosides (unpublished data). The THC-glycoside VB304 was the most significant detersive molecule, showing greatly increased foaming over saponins and SDS at the same concentration (Figure 5D). Interestingly, this foaming was more stable than that of other cannabosides, saponin, and SDS, and persisted for more than 8 hours.

Phytocannabinoids are known to act as agonists, antagonists, and inverse agonists of the human cannabinoid receptors, disrupting the endogenous cannabinoid signaling. This competition at the same active site indicates a functional and structural similarity between phytocannabinoids and endocannabinoids. Based on these similarities, the most well-characterized endocannabinoids were tested, and UGT76G1 was shown to glycosylate all endocannabinoids evaluated. A clear preference was observed for 2-AG and 1-AG compared with the ethanolamides AEA and DHEA. The weak glycosylation activity of UGT76G1 towards anandamide may also be due to non-optimal reaction conditions such as $\mathrm{pH}$, temperature, or specific buffer chemistry, or the ester group of the arachidonoylglycerols may be preferred over the amide group adjacent to the acceptor hydroxyl group of the ethanolamides. Further experimentation with additional endocannabinoids may shed light on the molecular components that contribute to binding within the UGT76G1 active site.

CBD has been published to degrade to THC, CBN, and quinone derivatives by light, heat, and acidic or basic conditions (for review Mechoulam and Hanus, 2002). Further studies have shown that orally administered CBD may degrade into the psychoactive THC in simulated 
gastric fluids containing 1\% SDS (Watanabe 2007, Merrick 2016). More recently, these in vitro results have been called into question as there is no in vivo evidence to support these claims (Russo 2017, Vitality Bio unpublished results). In the event that CBD is hydrolyzed to the psychoactive THC by gastric fluids, conjugating sugars to the free hydroxyl groups may protect cannabinoids from conditions that might otherwise produce unwanted cannabinoid byproducts within the stomach.

A growing body of evidence shows that glycosides are capable of acting as prodrugs and have direct therapeutic effects. One example of a class of glycoside pharmaceutical prodrugs is senna glycosides (Senokot, Ex-Lax), which are administered orally, followed by decoupling of the sugars in the large intestine by $\beta$-glucosidases secreted from intestinal microbiota (Hardcastle 1970). Site-specific delivery of steroid glycosides to the colon has also been demonstrated (Friend 1985, Friend 1984). Glycosylation of steroids enabled survival of stable bioactive molecules in the acidic stomach environment and delivery into the large intestine, where the aglycones were decoupled by glycosidases and absorbed into the systemic circulation. Colon-specific delivery of cannabosides and decoupling by local $\beta$-glucosidases may enable treatment of colon-specific disorders such as inflammatory bowel disease (IBD), including Crohn's disease and ulcerative colitis (Kunos 2004). In addition to facile delivery to the colon, $\beta$-glycosidases are also present universally in different tissues; therefore, delivery of cannabosides by methods that bypass the digestive tract and colon, such as intravenous or intranasal delivery, may enable delivery of cannabinoid aglycones to cells and tissues with adequate expression of glucosidases (Conchie 1959). Additionally, increasing the diversity and complexity of sugar attachments of cannabosides may provide altered distribution and pharmacokinetics in vivo, such as for extended- or delayed-release applications depending on the kinetics of glycoside decoupling. Glycoside prodrugs may thus enable site-specific and tissue-specific drug delivery, which would release simple glucose sugars upon prodrug decoupling. Cannabosides used as prodrugs could ultimately enable novel methods of delivering cannabinoids within the body, including the potential to limit the entry of psychoactive compounds into the bloodstream or brain through well-tolerated oral drug formulation and targeted delivery.

In the course of the present work, it was discovered that UGT76G1 from Stevia rebaudiana is capable of glycosylating a diverse range of substrates beyond steviol glycosides, including both 
aglycones and glycosides of multiple cannabinoid species. This promiscuous activity is in agreement with data published during the preparation of this manuscript showing that UGT76G1 may be active towards a wide range of substrates ranging from small aglycones to larger glycosides (Dewitte 2016).

In summary, this collection of novel cannabosides represents a greatly expanded class of cannabinoids with improved physicochemical properties and a wide variety of potential therapeutic applications. Further studies are currently underway to determine the safety and efficacy of these compounds for use as cannabinoid prodrugs.

\section{Materials and Methods:}

General Materials and Methods. Reverse-phase high-performance liquid chromatography (RP-HPLC) was performed on a Dionex 3000 LC system using a Phenomenex Kinetex $5 \mu m$ XB-C18 $100 \AA, 150 \times 4.6 \mathrm{~mm}$ column. Conditions were as follows: column temperature $30^{\circ} \mathrm{C}$, autosampler $5^{\circ} \mathrm{C}$, flow rate $1.0 \mathrm{~mL} / \mathrm{min}$, running time $25 \mathrm{~min}$, solvent $A$ acetonitrile, solvent $B$ water. Solvent A was started at 10\% for 4', then ramped to $99 \%$ over 10', held at $99 \%$ for 4', ramped down to $10 \%$ over 2', and held at 10\% for 5'. Detection at $202 \mathrm{~nm}$ provided the best information for all cannabinoids and cannabinoid glycosides. Cannabinoids and cannabinoid glycosides were identified using reference standards and the observation of a doublet peak at the cannabinoid absorbance maximum of $275 \mathrm{~nm}$. ${ }^{1} \mathrm{H}-\mathrm{NMR}$ spectroscopy was performed on a Bruker Avance II $400 \mathrm{MHz}$ instrument (Emery Pharma) with $\mathrm{CD}_{3} \mathrm{OD}$ as the solvent, using TopSpin acquisition and processing software. Liquid chromatography-electrospray ionization mass spectrometry (LC-ESI-MS) analysis was conducted on a Shimadzu LCMS 2010EV instrument (Emery Pharma). LC separation was performed using a Silia Chrom XDB C18 5um, 150A, 4.6X50 mm. The method was 12 minutes with a 5 to 95 water:acetonitrile gradient elution. Low-resolution MS was performed in both positive and negative modes. Acetic acid and formic acid were used as sample additives during analysis, and the injection volumes ranged from 5 to $20 \mu \mathrm{l}$.

Cloning, Expression, Purification, and Characterization of UGT76G1 and Os03g0702000p. A Stevia rebaudiana transcriptome library was created. mRNA was extracted from mature stevia 
leaves using a Spectrum Plant Total RNA extraction kit (Sigma Aldrich). Library preparation and next-gen sequencing were performed on an Illumina HiSeq2000 (Genewiz). Sequence reads were assembled using CLC Genomics Workbench 5.5.1 (Genewiz). The resulting transcriptome was queried with known Stevia UGT proteins via TblastN searches on a local BLAST server. The cDNA of UGT76G1 was amplified from mRNA via PCR utilizing the Bio-Rad iScript cDNA Synthesis Kit. Os03g0702000p was synthesized (IDT DNA) and PCR to amplify the genes of UGT76G1 and Os03g0702000p was performed utilizing primers listed in Supplementary S1. The primers were designed to add a 3' HQ tag to UGT76G1 and a 5' HQ tag to Os03g0702000p. Reaction mixtures included cDNA $(2 \mu \mathrm{l})$, forward and reverse primers (10 $\mu \mathrm{M}$ each), dNTPs (200 $\mu \mathrm{M})$, Phusion HF buffer (1X), and Phusion DNA Polymerase (0.5 $\mu \mathrm{l}, 1.0$ unit). The amplified sequences were inserted into the inducible pLATE11 vector (Thermo Scientific) using aLICator Ligation Independent Cloning and Expression System Kit 1. The resulting plasmids were used to transform E. coli BL21(DE3) competent cells (New England BioLabs). Positive transformants for each enzyme were confirmed by colony PCR using LIC forward and reverse primers.

Transformant cells for each enzyme were cultivated in 2xYT growth medium containing $100 \mu \mathrm{g} / \mathrm{ml}$ ampicillin while shaking at $20^{\circ} \mathrm{C}$ overnight. Then, $1.6 \mathrm{ml}$ of inoculum was added to flasks containing $250 \mathrm{ml}$ 2xYT growth medium with $100 \mu \mathrm{g} / \mathrm{ml}$ ampicillin. Cultures were grown to an optical density of 0.6 at $595 \mathrm{~nm}$. UGT76G1 and Os03g0702000p expression was induced by addition of isopropyl-1-b-D-thiogalactoside (IPTG) to a final concentration of $1 \mathrm{mM}$, followed by cultivation at $20^{\circ} \mathrm{C}$ while shaking for an additional 24 hours.

The cells were harvested by centrifugation at $8,000 \mathrm{~g}$ for $15 \mathrm{~min}$, then resuspended in Bead Wash Buffer containing $50 \mathrm{mM}$ potassium phosphate (pH 8.0) and $300 \mathrm{mM}$ sodium chloride $(\mathrm{NaCl})$. The cells were lysed by 2 passes through a microfluidizer, and the cellular debris was then removed by centrifugation. The supernatants were applied to Ni-NTA affinity columns (McLab) that had been equilibrated with Bead Wash Buffer. The columns were washed with 10 bed volumes of Wash Buffer containing 50 mM potassium phosphate (pH 8.0), $300 \mathrm{mM}$ $\mathrm{NaCl}$, and $2 \mathrm{mM}$ imidazole. The 3' HQ tagged UGT76G1 and 5' HQ tagged Os03g0702000p were eluted from the Ni-NTA columns with 10 bed volumes of Elution Buffer containing $50 \mathrm{mM}$ potassium phosphate (pH 8.0), $300 \mathrm{mM} \mathrm{NaCl}$, and $250 \mathrm{mM}$ imidazole. Sterile glycerol was added to each enzyme eluate to make a 50\% UGT76G1 and Os03g0702000p glycerol stock solution, which was stored at $-20^{\circ} \mathrm{C}$. Characterization and purity analysis of the fractions eluted with $250 \mathrm{mM}$ imidazole were performed by sodium dodecyl sulfate (SDS)-polyacrylamide gel 
electrophoresis (PAGE) using a 10\% gel. Protein concentrations were determined by Bradford assay.

\section{General Procedure for Glycosylation of Phytocannabinoids and Endocannabinoids with} UGT76G1. Glycosylation of selected phytocannabinoids and endocannabinoids by UGT76G1 was performed on a $200 \mu \mathrm{l}$ scale as proof of concept. The reactions were performed as follows. $1 \mu \mathrm{g}$ of the selected phytocannabinoid or endocannabinoid substrate $(1 \mu \mathrm{l}$ of a $1 \mathrm{mg} / \mathrm{ml}$ solution dissolved in methanol, $0.016 \mathrm{mM}$ final concentration) was added to $200 \mu \mathrm{l}$ of a reaction mixture containing $50 \mathrm{mM}$ potassium phosphate buffer ( $\mathrm{pH}$ 7.2), $3 \mathrm{mM}$ magnesium chloride $\left(\mathrm{MgCl}_{2}\right), 2.5$ $\mathrm{mM}$ uridine diphosphate glucose (UDPG) as the sugar donor, and $10 \mathrm{ml}$ of a $50 \%$ UGT76G1 glycerol stock solution ( $1.5 \mathrm{mg} / \mathrm{ml}$ stock solution, $1.44 \mu \mathrm{M}$ final protein concentration). The reaction mixtures were incubated at $28^{\circ} \mathrm{C}$ while shaking at $180 \mathrm{rpm}$ for $18 \mathrm{hrs}$. To stop the reaction, the reaction mixture was extracted three times with $200 \mu \mathrm{l}$ of ethyl acetate each time. The organic layers containing cannabinoid glycosides and any unreacted substrate were combined, and the ethyl acetate was removed under vacuum; then, the samples were redissolved in $100 \mu \mathrm{l}$ of $50 \% \mathrm{MeOH}$ by volume. Reaction products were analyzed by RP-HPLC on a Dionex 3000 LC system using a Phenomenex Kinetex $5 \mu \mathrm{m}$ XB-C18 100Å column (150 x $4.6 \mathrm{~mm}$ ). The conditions were as follows: injection volume of $50 \mu \mathrm{l}$; column temperature $30{ }^{\circ} \mathrm{C}$; autosampler $8{ }^{\circ} \mathrm{C}$; flow $1 \mathrm{ml} / \mathrm{min}$; running time $25 \mathrm{~min}$; solvent $A$ deionized water $\left(\mathrm{di}-\mathrm{H}_{2} \mathrm{O}\right)$, solvent $B$ acetonitrile (ACN), gradient elution 10-99\% ACN.

Glycosylation reactions for the phytocannabinoids CBD and THC were scaled up proportionally to $100 \mathrm{ml}$ to obtain an adequate amount of glycosylated products for characterization by LC-MS and ${ }^{1} \mathrm{H}$ NMR. UGT76G1 was deactivated and precipitated from the glycosylation reaction mixture by treatment at $95^{\circ} \mathrm{C}$ for 10 minutes. Clarified reaction mixture was obtained by centrifugation. Purification and separation of the glycosylated products is described below.

\section{Glycosylation of Phytocannabinoids and Endocannabinoids with Os03g0702000p.}

Glycosylation of selected phytocannabinoids and endocannabinoids by Os03g0702000p was performed on a $200 \mu \mathrm{l}$ scale as proof of concept. Os03g0702000p adds secondary glucose residues via $\beta-2-1$ connectivity from the primary glucose moiety; therefore, UGT76G1 was included in the reaction mixture to establish the primary glycosylation. The reactions were performed as follows. First, $1 \mu \mathrm{g}$ of the selected phytocannabinoid or endocannabinoid substrate ( $1 \mu \mathrm{l}$ of a $1 \mathrm{mg} / \mathrm{ml}$ solution dissolved in methanol, $0.016 \mathrm{mM}$ final concentration) was 
added to $200 \mu \mathrm{l}$ of a reaction mixture containing $50 \mathrm{mM}$ potassium phosphate buffer (pH 7.2), 3 $\mathrm{mM}$ magnesium chloride $\left(\mathrm{MgCl}_{2}\right), 2.5 \mathrm{mM}$ uridine diphosphate glucose (UDPG) as the sugar donor, $10 \mu \mathrm{l}$ of a $50 \%$ UGT76G1 glycerol stock solution $(1.5 \mathrm{mg} / \mathrm{ml}$ stock solution, $1.44 \mu \mathrm{M}$ final protein concentration), and $10 \mu \mathrm{l}$ of a $50 \%$ Os03g0702000p glycerol stock solution $(1.0 \mathrm{mg} / \mathrm{ml}$ stock solution, $0.962 \mu \mathrm{M}$ final protein concentration). The reaction mixtures were incubated at $28^{\circ} \mathrm{C}$ while shaking at $180 \mathrm{rpm}$ for $18 \mathrm{hrs}$. To stop the reaction, the reaction mixture was extracted three times with $200 \mu$ l of ethyl acetate each time. The organic layers containing cannabinoid glycosides and any unreacted substrate were combined and the ethyl acetate was removed under vacuum; then, the samples were redissolved in $100 \mu \mathrm{l}$ of $50 \% \mathrm{MeOH}$ by volume. The reaction products were analyzed by RP-HPLC on a Dionex 3000 LC system using a Phenomenex Kinetex $5 \mu \mathrm{m}$ XB-C18 $100 \AA$ column (150 x $4.6 \mathrm{~mm})$. The conditions were as follows: injection volume $50 \mu \mathrm{l}$, column temperature $30^{\circ} \mathrm{C}$, autosampler $8{ }^{\circ} \mathrm{C}$, flow $1 \mathrm{ml} / \mathrm{min}$, running time 25 min, solvent $A$ deionized water $\left(\mathrm{di}-\mathrm{H}_{2} \mathrm{O}\right)$, solvent $\mathrm{B}$ acetonitrile $(\mathrm{ACN})$, gradient elution $10-99 \%$ ACN.

Purification of CBD and THC Glycosides. CBD and THC glycosides were purified by C18 solid phase extraction. Hypersep C18 columns (Thermo) were prepared by hydration in methanol $(\mathrm{MeOH})$, followed by $50 \% \mathrm{MeOH}$, then rinsed with di-H2O. UGT76G1 was deactivated and precipitated from the glycosylation reaction mixture by treatment at $95{ }^{\circ} \mathrm{C}$ for 10 minutes; then, the clarified reaction mixture was passed through the column. The column was washed successively with 10 bed volumes of di-H2O,10\% $\mathrm{MeOH}$, and $30 \% \mathrm{MeOH}$. CBD Glycoside products were eluted with $60 \% \mathrm{MeOH}$ by volume, whereas $\mathrm{THC}$ glycosides were eluted with $80 \% \mathrm{MeOH}$ by volume. The purity of the glycoside mixtures was analyzed by RP-HPLC as described above.

Spectroscopic Analysis of Purified CBD Glycoside Mixture. CBD glycoside products produced in the glycosylation reaction with UGT76G1 were analyzed by liquid chromatography-electrospray ionization low-resolution mass spectrometry (LC/ESI-LRMS). The analysis was performed at Emery Pharma on a Shimadzu LC-MS 2010 EV instrument. The LC separation was performed on a $5 \mu \mathrm{m}$ Silia Chrom XDB C18 $150 \AA$ column $(50 \times 4.6 \mathrm{~mm})$. The running time was 12 min and a 5-95\% ACN elution gradient was utilized. LRMS was performed in positive mode with formic acid as a sample additive. The injection volume was $20 \mu$ l. Masses 
corresponding to the CBD aglycone up to the addition of 4 glucose residues were identified as follows:

CBD Aglycone $[\mathrm{M}+\mathrm{H}]^{+}\left(\mathrm{C}_{21} \mathrm{H}_{31} \mathrm{O}_{2}\right)$ Calcd: $m / z=315$. Found: $m / z=315$;

VB102/103 $[\mathrm{M}+\mathrm{H}]^{+}\left(\mathrm{C}_{27} \mathrm{H}_{41} \mathrm{O}_{7}\right)$ Calcd: $m / z=477$. Found: $m / z=477$;

VB104 $[\mathrm{M}+\mathrm{H}]^{+}\left(\mathrm{C}_{33} \mathrm{H}_{51} \mathrm{O}_{12}\right)$ Calcd: $m / z=639$. Found: $m / z=639$;

VB110 $[\mathrm{M}+\mathrm{H}]^{+}\left(\mathrm{C}_{33} \mathrm{H}_{51} \mathrm{O}_{12}\right)$ Calcd: $m / z=639$. Found: $m / z=639$;

VB112/118 $[\mathrm{M}+\mathrm{H}]^{+}\left(\mathrm{C}_{39} \mathrm{H}_{61} \mathrm{O}_{17}\right)$ Calcd: $m / z=801$. Found: $m / z=801 .[\mathrm{M}+\mathrm{K}+\mathrm{H}]^{+}\left(\mathrm{C}_{39} \mathrm{H}_{61} \mathrm{O}_{17} \mathrm{~K}\right)$

Calcd: $m / z=420$. Found: $m / z=420$. $\left[\mathrm{M}+\mathrm{ACN}+\mathrm{H}_{2} \mathrm{O}+\mathrm{H}^{+}\left(\mathrm{C}_{41} \mathrm{H}_{63} \mathrm{NO}_{17}\right)\right.$ Calcd: $m / z=860$.

Found: $m / z=860 ;$ and

VB119 $[\mathrm{M}+\mathrm{H}]^{+}\left(\mathrm{C}_{45} \mathrm{H}_{71} \mathrm{O}_{22}\right)$ Calcd: $m / z=964$. Found: $m / z=964 .\left[\mathrm{M}+\mathrm{H}_{2} \mathrm{O}+\mathrm{H}\right]^{+}\left(\mathrm{C}_{45} \mathrm{H}_{73} \mathrm{O}_{18}\right)$

Calcd: $m / z=983$. Found: $m / z=983$.

\section{Spectroscopic Analysis of Purified and Separated CBD Glycoside VB104}

${ }^{1} \mathrm{H}-\mathrm{NMR}$ was obtained on a Bruker Avance II $400 \mathrm{MHz}$ instrument using TopSpin acquisition and SpinWorks 4 processing software. $1 \mathrm{H}$ NMR $\left(\mathrm{CD}_{3} \mathrm{OD}\right): \delta 0.81\left(\mathrm{t}, 3 \mathrm{H}, \mathrm{H}_{5^{\prime \prime}}\right), 1.19\left(\mathrm{~m}, 4 \mathrm{H}, \mathrm{H}_{3^{\prime \prime}}\right.$, $\left.\mathrm{H}_{4^{\prime \prime}}\right), 1.46\left(\mathrm{t}, 2 \mathrm{H}, \mathrm{H}_{2^{\prime \prime}}\right), 1.54\left(\mathrm{~s}, 3 \mathrm{H}, \mathrm{H}_{10}\right), 1.59\left(\mathrm{~s}, 3 \mathrm{H}, \mathrm{H}_{7}\right), 1.65\left(\mathrm{~m}, 2 \mathrm{H}, \mathrm{H}_{5}\right), 1.89\left(\mathrm{~d}, 1 \mathrm{H}, \mathrm{H}_{4}\right), 2.41$ $\left(\mathrm{t}, 2 \mathrm{H}, \mathrm{H}_{1^{\prime \prime}}\right), 2.95\left(\mathrm{~m}, 1 \mathrm{H}, \mathrm{H}_{6}\right), 3.18-3.70\left(\mathrm{~m}, 12 \mathrm{H}, \mathrm{H}_{\mathrm{glu}}\right), 3.78\left(\mathrm{dd}, 2 \mathrm{H}, \mathrm{H}_{1}\right), 3.94\left(\mathrm{~d}, 1 \mathrm{H}, \mathrm{H}_{2}\right), 4.31$ $\left(\mathrm{m}, 1 \mathrm{H}, \mathrm{H}_{9 \mathrm{cis}}\right), 4.42\left(\mathrm{~d}, 1 \mathrm{H}, \mathrm{H}_{\text {9trans }}\right), 4.50\left(\mathrm{~d}, 1 \mathrm{H}, \mathrm{H}_{\beta-1^{*}}\right), 5.18\left(\mathrm{~s}, 1 \mathrm{H}, \mathrm{H}_{\mathrm{a}-1^{*}}\right), 6.19\left(\mathrm{~s}, 1 \mathrm{H}, \mathrm{H}_{5^{\prime}}\right), 6.36(\mathrm{~s}$, $\left.1 \mathrm{H}, \mathrm{H}_{3}\right)$.

\section{Spectroscopic Analysis of Purified and Separated CBD Glycoside VB110}

${ }^{1} \mathrm{H}-\mathrm{NMR}$ was obtained on a Bruker Avance II $400 \mathrm{MHz}$ instrument using TopSpin acquisition and SpinWorks 4 processing software. $1 \mathrm{H}$ NMR $\left(\mathrm{CD}_{3} \mathrm{OD}\right): 0.79\left(\mathrm{t}, 3 \mathrm{H}, \mathrm{H}_{5^{\prime \prime}}\right), 1.16\left(\mathrm{~m}, 5 \mathrm{H}, \mathrm{H}_{3^{\prime \prime}}\right.$, $\left.\mathrm{H}_{4^{\prime \prime}}\right), 1.47\left(\mathrm{~m}, 3 \mathrm{H}, \mathrm{H}_{2^{\prime \prime}}, \mathrm{H}_{10}\right), 1.59\left(\mathrm{~s}, 1 \mathrm{H}, \mathrm{H}_{7}\right), 1.65\left(\mathrm{~m}, 1 \mathrm{H}, \mathrm{H}_{5}\right), 1.91\left(\mathrm{~m}, 1 \mathrm{H}, \mathrm{H}_{5}\right), 2.43\left(\mathrm{t}, 1 \mathrm{H}, \mathrm{H}_{1^{\prime \prime}}\right)$, $2.99\left(\mathrm{~m}, 1 \mathrm{H}, \mathrm{H}_{6}\right), 3.16-3.67\left(\mathrm{~m}, 11 \mathrm{H}, \mathrm{H}_{\mathrm{glu}^{*}}\right), 3.77\left(\mathrm{dd}, 1 \mathrm{H}, \mathrm{H}_{1}\right), 4.07\left(\mathrm{~m}, 1 \mathrm{H}, \mathrm{H}_{2}\right), 4.29(\mathrm{~m}, 1 \mathrm{H}$, $\left.\mathrm{H}_{9 \text { cis }}\right), 4.45\left(\mathrm{~d}, 1 \mathrm{H}, \mathrm{H}_{9 \text { trans }}\right), 4.49\left(\mathrm{~d}, 1 \mathrm{H}, \mathrm{H}_{\beta-1^{*}}\right), 5.23\left(\mathrm{~s}, 1 \mathrm{H}, \mathrm{H}_{\mathrm{\alpha}-1^{*}}\right), 6.59\left(\mathrm{~d}, 1 \mathrm{H}, \mathrm{H}_{3^{\prime}}, \mathrm{H}_{5^{\prime}}\right)$

Spectroscopic Analysis of Purified THC Glycoside Mixture. THC glycoside products produced in the glycosylation reaction with UGT76G1 were analyzed by liquid chromatography-electrospray ionization low-resolution mass spectrometry (LC/ESI-LRMS). The analysis was performed at Emery Pharma on a Shimadzu LC-MS 2010 EV instrument. The LC separation was performed on a $5 \mu \mathrm{m}$ Silia Chrom XDB C18 $150 \AA$ column $(50 \times 4.6 \mathrm{~mm})$. The running time was $12 \mathrm{~min}$ and a 5-95\% ACN elution gradient was utilized. LRMS was performed 
in positive mode with formic acid as a sample additive. The injection volume was $20 \mu \mathrm{l}$. Masses corresponding with the THC aglycone along with glycosides conjugated with 2 and 3 glucose residues were identified as follows:

THC Aglycone $[\mathrm{M}+\mathrm{H}]^{+}\left(\mathrm{C}_{21} \mathrm{H}_{31} \mathrm{O}_{2}\right)$ Calcd: $m / z=315$. Found: $m / z=315$;

VB304 $[\mathrm{M}+\mathrm{H}]^{+}\left(\mathrm{C}_{33} \mathrm{H}_{51} \mathrm{O}_{12}\right)$ Calcd: $m / z=639$. Found: $m / z=639$; and

VB308 $[\mathrm{M}+\mathrm{H}]^{+}\left(\mathrm{C}_{39} \mathrm{H}_{61} \mathrm{O}_{17}\right)$ Calcd: $m / z=801$. Found: $m / z=801 .[\mathrm{M}+\mathrm{Na}]^{+}\left(\mathrm{C}_{39} \mathrm{H}_{60} \mathrm{O}_{17} \mathrm{Na}\right)$ Calcd: $m / z=823$. Found: $m / z=823 .[\mathrm{M}+\mathrm{K}+\mathrm{H}]^{+}\left(\mathrm{C}_{39} \mathrm{H}_{61} \mathrm{O}_{17} \mathrm{~K}\right)$ Calcd: $m / z=420$. Found: $m / z=420$.

cLogP Calculations. cLogP (logarithm of the partition coefficient between n-octanol and water) calculations were performed using ChemDraw Ultra (CambridgeSoft Corp., Cambridge, MA).

Empirical Determination of Solubility and Detersiveness. 5 milligrams of cannabosides and control chemicals were weighed out and added to $2.0 \mathrm{ml}$ glass HPLC vials. 500 microliters of sterile distilled water was added and the resulting solution was mixed by pipetting and gentle swirling. Solutions were then vortexed using a laboratory vortexer for 1 minute (VWR Vortex Genie, setting 10). Images were taken at the indicated steps and cropped using GNU Image Manipulation Program, and assembled using Inkscape.

\section{References:}

Aizpurua-Olaizola, O, et al. (2017) Targeting the endocannabinoid system: future therapeutic strategies. Drug Discovery Today. 22(1): DOI: 10.1016/j.drudis.2016.08.005.

Atakan, Z. (2012). Cannabis, a complex plant: different compounds and different effects on individuals. Ther Adv Psychopharmacol. 2(6):241-254.

Bartzokis G. (2004). Age-related myelin breakdown: a developmental model of cognitive decline and Alzheimer's disease. Neurobiology of Aging. 25:5-18.

Bisogno T, et al. (2001) Molecular targets for cannabidiol and its synthetic analogues: effect on vanilloid VR1 receptors and on the cellular uptake and enzymatic hydrolysis of anandamide. British Journal of Pharmacology. 134, 845-852.

Chen $Q$, et al. (2009). Synthesis, in vitro and in vivo characterization of glycosyl derivatives of ibuprofen as novel prodrugs for brain drug delivery. J Drug Targeting. 17(4):318-328.

Conchie J., Findlay J., Levvy GA. (1958). Mammalian Glycosidases, Distribution in the body. Biochem J. 71(2):318-325. 
De Petrocellis L, et al. (2011) Effects of cannabinoids and cannabinoid-enriched Cannabis extracts on TRP channels and endocannabinoid metabolic enzymes. British Journal of Pharmacology. 163, 1479-1494.

Dewitte G, et al. (2016) Screening of Recombinant Glycosyltransferases Reveals the Broad acceptor Specificity of Stevia UGT-76G1. Journal of Biotechnology. Accepted Manuscript, DOI: http://dx.doi.org/doi:10.1016/j.jbiotec.2016.06.034.

DiPatrizio N.V. (2016) Endocannabinoids in the Gut. Cannabis and Cannabinoid Research. $1(1): 67-77$.

Friend DR., Chang GW. (1984). A Colon-Specific Drug-Delivery System Based on Drug Glycosides and the Glycosidases of the Colonic Bacteria. J Med Chem. 27:261-266. Friend DR., Chang GW. (1985). Drug Glycosides: Potential Prodrugs for Colon-Specific Drug Delivery. J Med Chem. 28:51-57.

Gomez O., Arevalo-Martin A., Garcia-Ovejero D., Ortega-Gutierrez S., Cisneros JA., Almazan G, Sanchez-Rodriguez MA., Molina-Holgado F., Molina-Holgado E. (2010). The Constitutive Production of the Endocannabinoid 2-Arachidonoylglycerol Participates in Oligodendrocyte Differentiation. Glia. 58:1913-1927.

Guex, N., Peitsch, M.C. Schwede, T. (2009). Automated comparative protein structure modeling with SWISS-MODEL and Swiss-PdbViewer: A historical perspective. Electrophoresis, 30(S1), S162-S173.

Hardcastle, JD., Wilkins, JL. (1970). The action of sennosides and related compounds on human colon and rectum. Gut. 11:1038-1042.

Hasenoehrl, C., Taschler, U., Storr, M., Schicho, R. (2016). The gastrointestinal tract - a central organ of cannabinoid signaling in health and disease. Neurogastroenterology \& Motility. 28(12):1765-1780.

Hryciw DH, McAinch AJ. (2016). Cannabinoid receptors in the kidney. Curr Opin Nephrol Hypertens. 25(5):459-464.

Iuvone T., Esposito G., De Filippis D., Scuderi C., Steardo L. (2009). Cannabidiol: a promising drug for neurodegenerative disorders? CNS Neurosci Ther. 15(1):65-75.

Jarh, P., Pate DW., Brenneisen R., Jarvinen T. (1998). Hydroxypropyl-beta-cyclodextrin and its combination with hydroxypropyl-methylcellulose increases aqueous solubility of delta9-tetrahydrocannabinol. Life Sci. 63(26):PL381-384.

Jiang R, et al. (2011) Identification of cytochrome P450 enzymes responsible for metabolism of cannabidiol by human liver microsomes. Life Sciences. 89, 165-170. 
Kiefer F, Arnold K, Künzli M, Bordoli L, Schwede T (2009). The SWISS-MODEL Repository and associated resources. Nucleic Acids Res. 37, D387-D392.

Kren V (2008) Glycoside vs. Aglycon: The Role of Glycosidic Residue in Biologic Activity. Glycoscience. pp2589-2644.

Kren V, Rezanka T (2008) Sweet antibiotics - the role of glycosidic residues in antibiotic and antitumor activity and their randomization. FEMS Microbiol Rev. 32, 858-889.

Kunos, G., Pacher, P. (2004). Cannabinoids cool the intestine. Nat Med. 10(7):678-679.

Leo A, Russo E, Elia M. 2016. Cannabidiol and epilepsy: Rationale and therapeutic potential. Pharmacol Res. 107:85-92.

Li S., Li W., Xiao Q., Xia Y. (2012). Transglycosylation of stevioside to improve the edulcorant quality by lower substitution using cornstarch hydrolyzate and CGTase. J Food Chem. 138(2013):2064-2069.

Mackie, K. (2008). Cannabinoid receptors: where they are and what they do. Journal of Neuroendocrinology. 20 (Supp. 1): 10-14

Martin, B. R., et al. (2006) Pharmacological Characterization of Novel Water-Soluble Cannabinoids. The Journal of Pharmacology and Experimental Therapeutics. 318(3): 1230-1239.

Mazur A., et al. (2009). Characterization of Human Hepatic and Extrahepatic UDP-Glucuronosyltransferase Enzymes Involved in the Metabolism of Classic Cannabinoids. Drug Metabolism and Disposition. 37(7):1496-1504.

Mecha M., Torrao AS., Mestre L., Carrillo-Salinas FJ., Mechoulam R., Guaza C. (2012). Cannabidiol protects oligodendrocyte progenitor cells from inflammation-induced apoptosis by attenuating endoplasmic reticulum stress. Cell Death and Disease. 3(e331).

Mechoulam, R, Hanus, L. (2002). Cannabidiol: an overview of some chemical and pharmacological aspects. Part I: chemical aspects. Chemistry and Physics of Lipids. 121:35-43.

Mechoulam R., Parker LA., Gallily R. (2002). Cannabidiol: An Overview of Some Pharmacological Aspects. 42(S1):11S-19S.

Merrick, J., et al. (2016). Identification of Psychoactive Degradants of Cannabidiol in Simulated Gastric and Physiological Fluid. Cannabis and Cannabinoid Res. 1.1:102-112.

Mighdoll MI., Tao R., Kleinman JE., Hyde TM. (2015). Myelin, myelin-related disorders, and psychosis. Schizophr Res. 161(1):85-93. 
Molina-Holgado E., Vela JM., Arevalo-Martin A., Almazan G., Molina-Holgado F., Borrell J., Guaza C. (2002). Cannabinoids Promote Oligodendrocyte Progenitor Survival:

Involvement of Cannabinoid Receptors and Phosphatidylinositol-3-Kinase/Akt Signaling. J. Neurosci. 22(22):9742-9753.

Noguchi A, et al. (2009). Identification of an inducible glucosyltransferase from Phytolacca americana L. cells that are capable of glucosylating capsaicin. Plant Biotechnology. 26, 285-292.

Pacher P, et al. (2006) The endocannabinoid system as an emerging target of pharmacotherapy. Pharmacology Review. 58(3), 389-462.

Porter, BE., Jacobson, C. 2013. Report of a parent survey of cannabidiol-enriched cannabis use in pediatric treatment-resistant epilepsy. Epilepsy Behav. 29(3):574-577.

Richman A., Swanson, A., Humphrey T., Chapman R., McGarvey B., Pocs R., Brandle J. (2005). Functional genomics uncovers three glucosyltransferases involved in the synthesis of the major sweet glucosides of Stevia rebaudiana. Plant J. 41(1):56-67.

Russo E., Guy, GW. (2006) A tale of two cannabinoids: the therapeutic rationale for combining tetrahydrocannabinol and cannabidiol. Medical Hypotheses. 66(2):234-46.

Russo E. (2017). Cannabidiol Claims and Misconceptions. Trends in Pharmacological Sci. (online).

Scully (2007). Cannabis; Adverse effects from an oromucosal spray. British Dental Journal. 203:E12.

Slavic S., et al. (2013). Cannabinoid receptor 1 inhibition improves cardiac function and remodelling after myocardial infarction and in experimental metabolic syndrome. J Mol Med (Berl). 91(7):811-23.

Tanaka H., et al. (1993). Cannabis, 21. ${ }^{1}$ Biotransformation of cannabinol to its glycosides by in vitro plant tissue. Journal of Natural Products. 56(12):2068-2072.

Tanaka H., et al. (1996). Cannabis 25, biotransformation of cannabidiol and cannabidiolic acid by Pinellia ternata tissue segments. Plant Cell Reports. 15:819-823.

Terao J., Murota K., Kawai Y. (2011). Conjugated quercetin glucuronides as bioactive metabolites and precursors of aglycone in vivo. Food Function. 2:11-17.

Thomas A., et al. (2007) Cannabidiol displays unexpectedly high potency as an antagonist of $\mathrm{CB}_{1}$ and $\mathrm{CB}_{2}$ receptor agonists in vitro. British Journal of Pharmacology. 150, 613-623. Touw, M. (1981). The religious and medicinal uses of Cannabis in China, India, and Tibet. J Psychoactive Drugs. 13(1). 
US Patent 8,410,064 B2. 2013. Classical cannabinoid metabolites and methods of use thereof.

US Patent 8,227,627 B2. 2012. Prodrugs of tetrahydrocannabinol, compositions comprising prodrugs of tetrahydrocannabinol and methods of using the same.

Watanabe K, et al. (1998) Distribution and characterization of anandamide amidohydrolase in mouse brain and liver. Life Sciences. 62(14), 1223-1229.

Watanabe, K, et al. (2007). Conversion of cannabidiol to delta-9-tetrahydrocannabinol and related cannabinoids in artificial gastric juice, and their pharmacological effects in mice. Forensic Toxicology. 25(1):16-21.

Welling, M.T., Liu, L., Shapter, T. et al.(2016). Characterisation of cannabinoid composition in a diverse Cannabis sativa L. germplasm collection. Euphytica. 208: 463. doi:10.1007/s10681-015-1585-y, WO2009018389 A4. 2009. Prodrugs of cannabidiol, compositions comprising prodrugs of cannabidiol and methods of using the same.

WO2012011112 A1. 2011. Non psychoactive cannabinoids and uses thereof. WO2013022989 A2. 2013. Recombinant production of steviol glycosides.

WO2014108899 A1. 2014. Fluorinated CBD compounds, compositions and uses thereof. Yamaori S, et al. (2011) Potent inhibition of human cytochrome P450 3A isoforms by cannabidiol: Role of phenolic hydroxyl groups in the resorcinol moiety. Life Sciences, 88, 730-736.

Zuardi AW, et al. (2012). A Critical Review of the Antipsychotic Effects of Cannabidiol: 30 Years of a Translational Investigation. Current Pharmaceutical Design, 18, 5131-5140.

\section{Conflict of Interest Statement:}

The authors disclose that they are employees of Vitality Biopharma Inc. and have filed patents on the material described herein.

\section{Figure Captions:}

\section{Figure 1: UGT76G1 from Stevia rebaudiana glycosylates cannabidiol (CBD)}

Cannabidiol was reacted with UGT76G1 in vitro and the reaction was observed by HPLC (A). The control reaction contained all reagents but lacked only the sugar donor UDPG (solid linetrace). Depletion of CBD was observed upon addition of UDPG, and major product peaks were present at 8.75', 9.0', 10.3', and 10.7' (dotted linetrace). CBD displayed a broad 
absorbance doublet centered at $275 \mathrm{~nm}$ (solid black line) that is maintained for VB104 (gray line) and shifted for VB110 (dotted line)(B). Structures of CBD and CBD-glycosides as determined by stepwise glycosylation reactions, LCMS-ESI-UV, and H-NMR (C). Product glycosides are organized by the total number of glycosylations present.

Figure 2: Secondary glycosylation with UGT76G1 and Os03g0702000p

CBD-glycoside VB104 was partially purified and further reacted with UGT76G1 (A). The control reaction contained no UDPG (solid linetrace), with the substrate containing VB104 (10.7') and a small VB110 peak (9.0'). Upon addition of UDPG, a decrease was seen in VB104 (dotted linetrace), as well as increases in VB110 and two minor peaks at 10.32' and 10.39'. A proposed biosynthetic pathway from VB104 to VB110 is depicted (B). Additionally, partially purified VB104 was further reacted with Os03g0702000p (C), with inputs as in Figure 2A (solid linetrace). Upon addition of UDPG, VB104 and VB110 decreased in amount, and new peaks were observed at 9.94', 10.27', and 10.31' (dotted linetrace).

\section{Figure 3: UGT76G1 glycosylates additional phytocannabinoids}

Cannabidivarin (CBDV) was reacted with UGT76G1 in vitro and the reaction was observed by HPLC (A). The control reaction contained all reagents but lacked only the sugar donor UDPG (solid linetrace). Depletion of CBDV (12.75') was observed upon addition of UDPG, and major product peaks were present at 8.52', 9.7', and 10.0' (dotted linetrace). $\Delta 9$-Tetrahydrocannabinol (THC) was reacted with UGT76G1 in vitro and the reaction was observed by HPLC (B). The control reaction contained all reagents but lacked only the sugar donor UDPG (solid linetrace). Depletion of THC (14.52') was observed upon addition of UDPG, and major product peaks were present at 10.64', 10.93', 11.68', and 12.48' (dotted linetrace). Cannabinol (CBN) was reacted with UGT76G1 in vitro and the reaction was observed by HPLC (C). The control reaction contained all reagents but lacked only the sugar donor UDPG (solid linetrace). Depletion of CBN (14.17') was observed upon addition of UDPG, and major product peaks were present at 10.85', 11.48', and 12.23' (dotted linetrace). Structures of THC and THC-glycosides as determined by stepwise glycosylation reactions, and LCMS-ESI-UV (D). Product glycosides are organized by the total number of glycosylations present.

Figure 4: UGT76G1 glycosylates endocannabinoids 
Anandamide (AEA) was reacted with UGT76G1 in vitro and the reaction was observed by HPLC (A). The control reaction contained all reagents but lacked only the sugar donor UDPG (solid linetrace). Depletion of AEA (13.85') was observed upon addition of UDPG, and the major product peak was present at 12.47' (dotted linetrace). A 9:1 mixture of 2-arachidonoyl-glycerol $(2 A G)$ and 1-arachidonoyl-glycerol (1AG) was reacted with UGT76G1 in vitro and the reaction was observed by HPLC (B). The control reaction contained all reagents but lacked only the sugar donor UDPG (solid linetrace). Depletion of 2AG (14.31') and 1AG (14.18') was observed upon addition of UDPG and major product peaks were present at 11.39', 11.70', 11.77', 11.89', 11.97', 12.51', 12.92', 13.07', and 13.32' (dotted linetrace). Synaptamide (DHEA) was reacted with UGT76G1 in vitro and the reaction was observed by HPLC (C). The control reaction contained all reagents but lacked only the sugar donor UDPG (solid linetrace). Depletion of DHEA (13.75') was observed upon addition of UDPG, and the major product peak was present at 12.42' with minor peaks at 10.09', and 10.21' (dotted linetrace). (D). Endocannabinoid substrates of UGT76G1.

Table 1: Physicochemical properties of cannabosides, cannabinoids, and cannabinoid-metabolites

The RP-HPLC retention times were determined empirically, and the ClogP values were calculated in silico for a subset of cannabosides, cannabinoids, and cannabinoid-metabolites.

\section{Figure 5: Physicochemical properties of cannabosides}

ClogP values for select cannabosides, cannabinoids, and cannabinoid-metabolites from Table 1 were plotted against RP-HPLC retention times to assess linearity (A). The $C 18$ retention times for cannabinoids (filled diamonds), cannabosides (open-diamonds), and cannabinoid-metabolites (filled circles) were plotted against ClogP values from Table 1 (A). Linear regression was performed on all data points $(R 2=0.9455)$ and plotted as the line in $(A)$. Aqueous solubility and detersive properties are displayed (B, C, and D). Solids in vials (B), hydrated and mixed by pipetting at $1 \%$ in water (C), and following 1 minute of vortexing (D). Vials are as follows: 1. Water, 2. CBD, 3. VB104, 4. VB110, 5. VB304, 6, Saponin, 7. SDS, and 8. Sucrose.

\section{Supplemental Figure 1: Phylogenetic tree of UGTs from Stevia rebaudiana}


UGT protein sequences were mined from a Stevia transcriptome and aligned using MUSCLE in UGENE (v1.9.8). The phylogenetic tree was assembled using PHYLIP neighbor joining with Jones-Taylor-Thornton distance matrix model. Individual distances were omitted for figure clarity.

\section{Supplemental Figure 2: LC-ESI-MS for cannabosides}

Figure 2. Mass spectra of cannabinoid glycosides generated by incubation with UGT76G1 and UDPG, where $\mathrm{gX}$ represents glucose plus the number of sugars attached to the parent compound. (A) Purified CBD glycoside mixture. (B) Separated CBD glycoside VB104.

(C) Separated CBD glycoside VB110. (D) Purified THC glycoside mixture.

\section{Supplemental Figure 3: Molecular superpositioning of UGT76G1 substrates}

A prototypical substrate of UGT76G1 from the stevia biosynthetic pathway, Rebaudioside E (RebE, in black), was overlaid with CBD (green) in a manner that would facilitate glycosylation of the 6'-OH group (A). RebE (black) was overlaid with CBD (green) in a manner that would facilitate glycosylation of the 2'-OH group (B).

\section{Supplemental Figure 4: Direct glycosylation model of CBD by UGT76G1}

A direct glycosylation model for the biosynthesis of CBD-glycosides by UGT76G1. CBD retains a rigid conformation and does not rotate around the C1' axis. Glycosylations are established as the overall substrate re-positions the recipient hydroxyls towards the enzyme catalytic site.

\section{Supplemental Figure 5: Rotational glycosylation model of CBD by UGT76G1}

A rotational model for the biosynthesis of CBD-glycosides by UGT76G1. After establishing one or two glycosylations on one of the hydroxyl groups of CBD, the rotational freedom along the C1' axis allows the resorcinol ring to rotate and swing the second hydroxyl group towards the catalytic site of UGT76G1.

\section{Supplemental Figure 6: Oligonucleotide sequences}

Primers used for the amplification and cloning of UGT76G1 and Os03g0702000. 
bioRxiv preprint doi: https://doi.org/10.1101/104349; this version posted January 30, 2017. The copyright holder for this preprint (which was not certified by peer review) is the author/funder, who has granted bioRxiv a license to display the preprint in perpetuity. It is made available under Hardman et al. 2017. Figure 1ạCC-BY-NC-ND 4.0 International license.
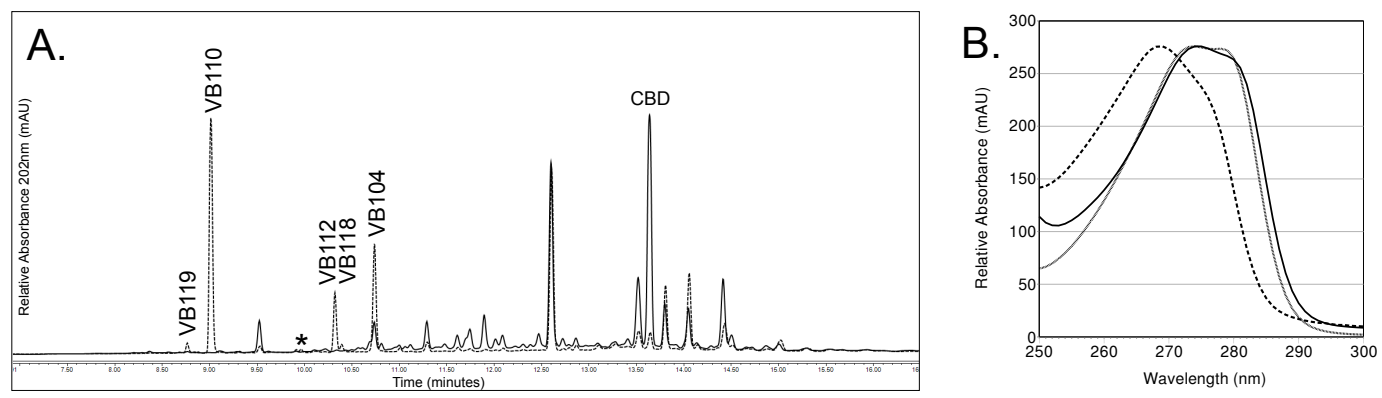

C.
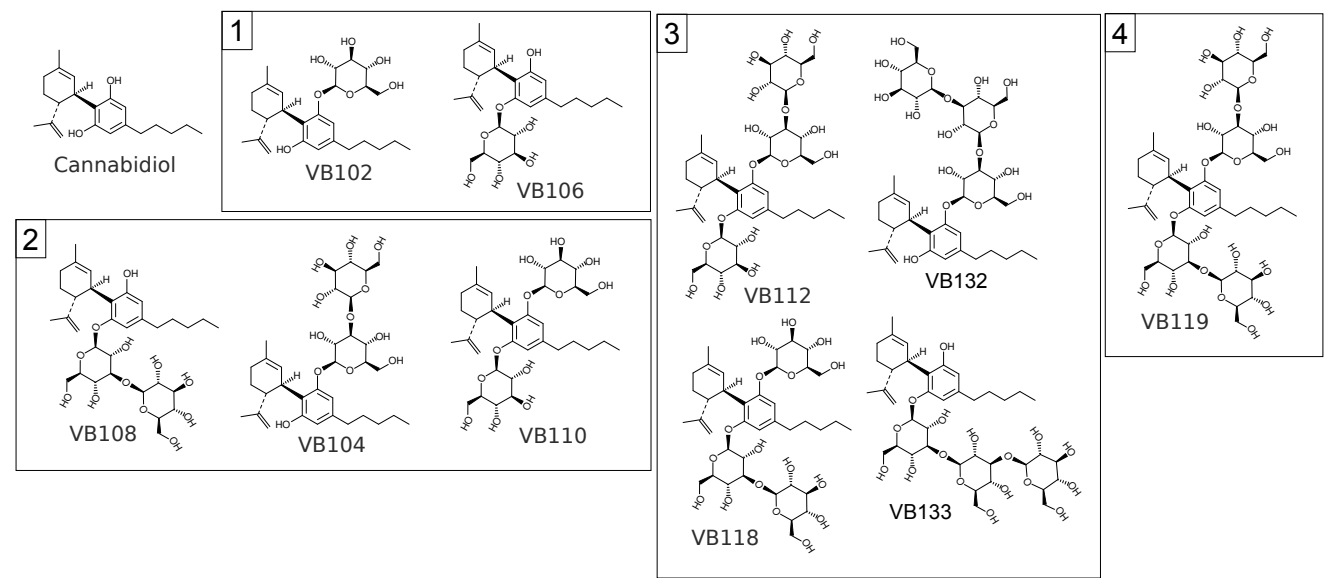
bioRxiv preprint doi: https://doi.org/10.1101/104349; this version posted January 30, 2017. The copyright holder for this preprint (which was not

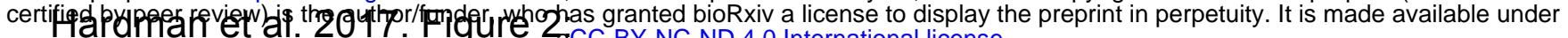

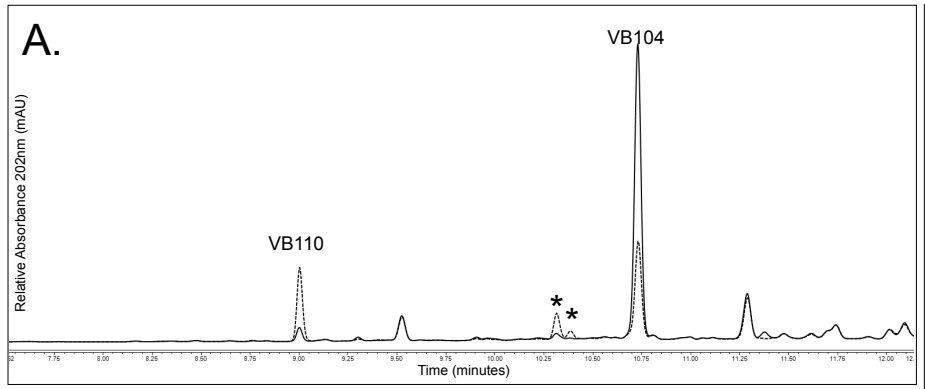

C.

VB104

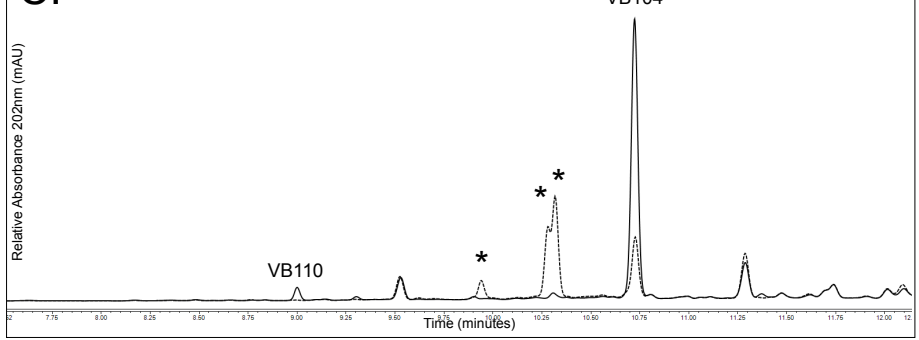

B.

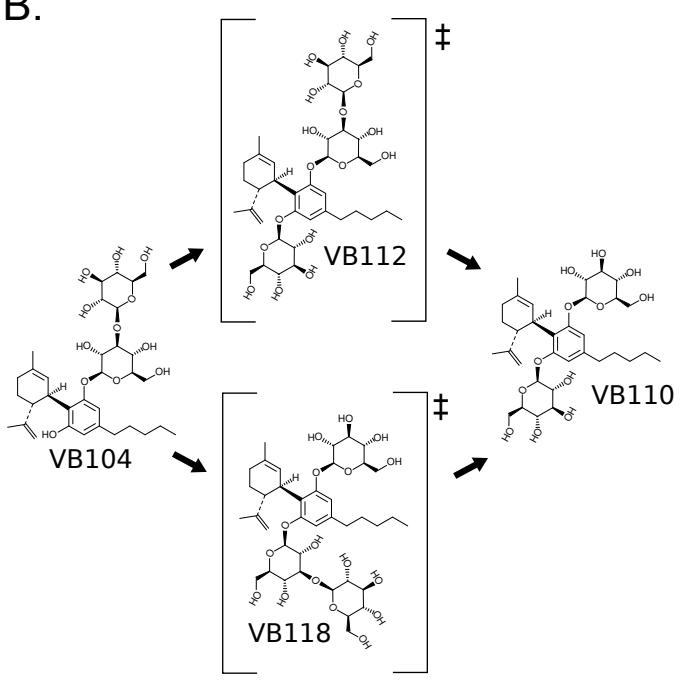


bioRxiv preprint doi: https://doi.org/10.1101/104349; this version posted January 30, 2017. The copyright holder for this preprint (which was not certified by peer review) is the author/funder, who has granted bioRxiv a license to display the preprint in perpetuity. It is made available under Hardman et al. 2017. Figure 3 .
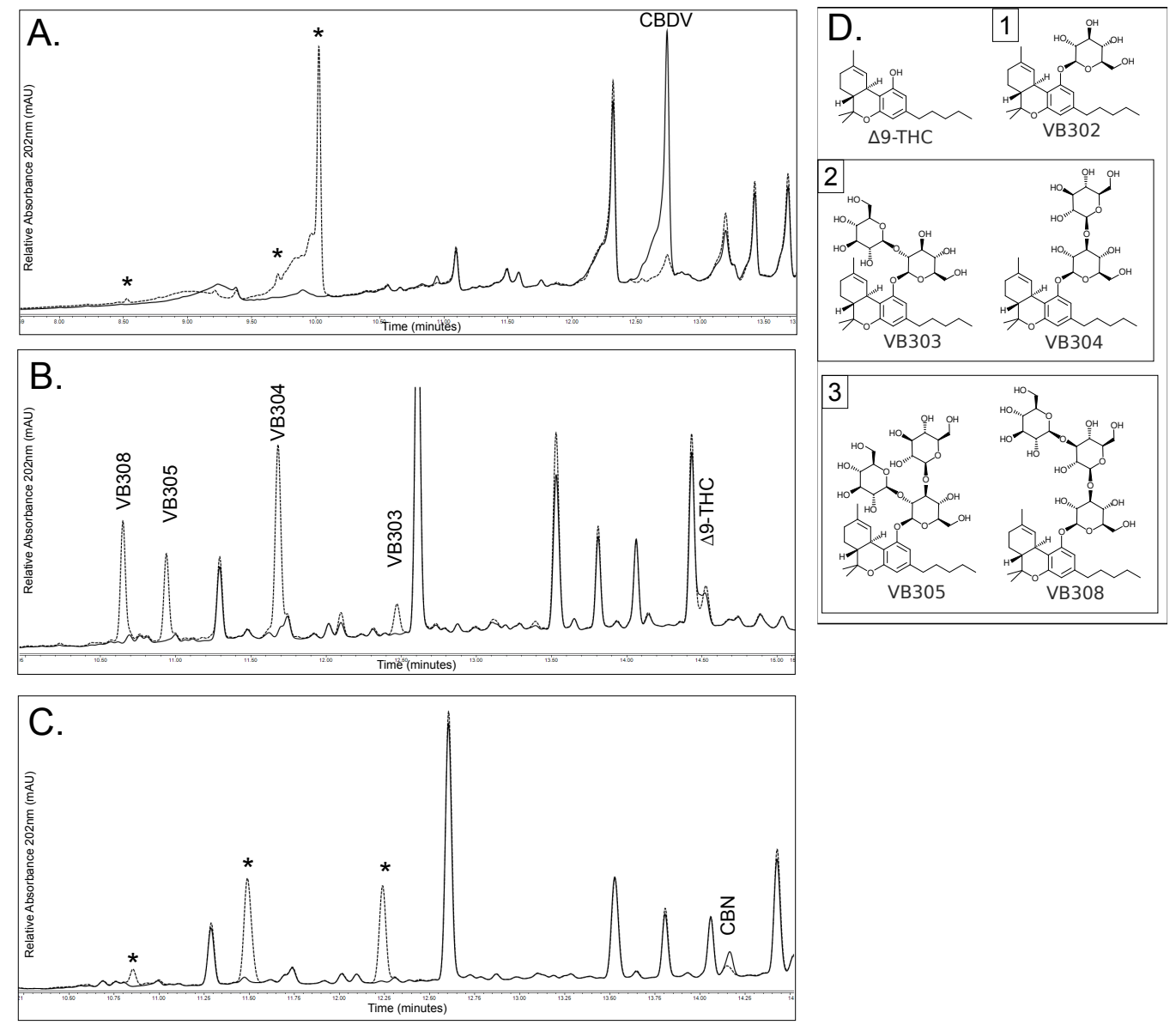
bioRxiv preprint doi: https://doi.org/10.1101/104349; this version posted January 30, 2017. The copyright holder for this preprint (which was not certified by peer review) is the author/funder, who has granted bioRxiv a license to display the preprint in perpetuity. It is made available under Hardman et al. 2017. Figure 4:
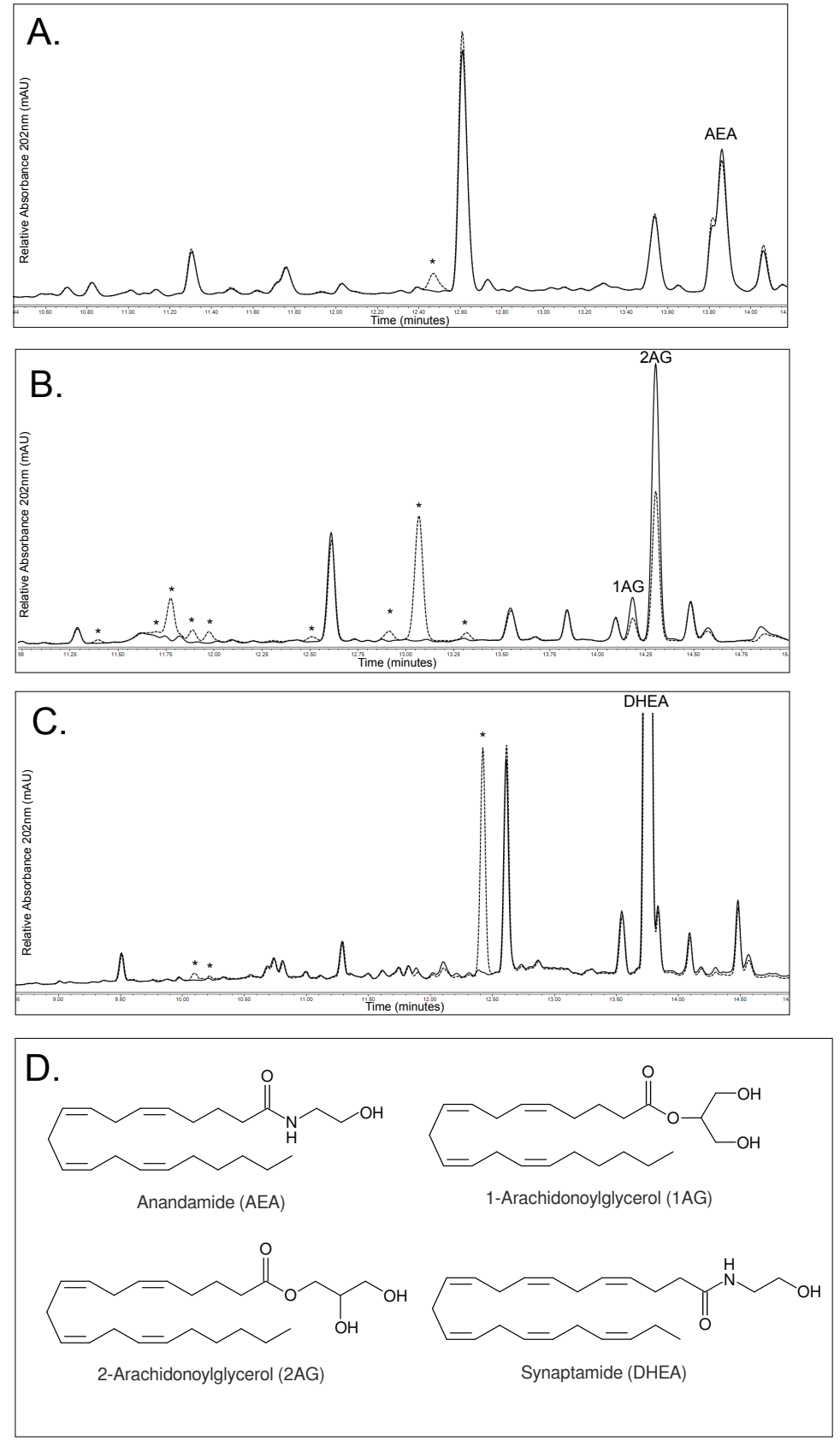
bioRxiv preprint doi: https://doi.org/10.1101/104349; this version posted January 30, 2017. The copyright holder for this preprint (which was not certified by peer review) is the author/funder, who has granted bioRxiv a license to display the preprint in perpetuity. It is made available under Hardman et al. 2017. Figure 5:
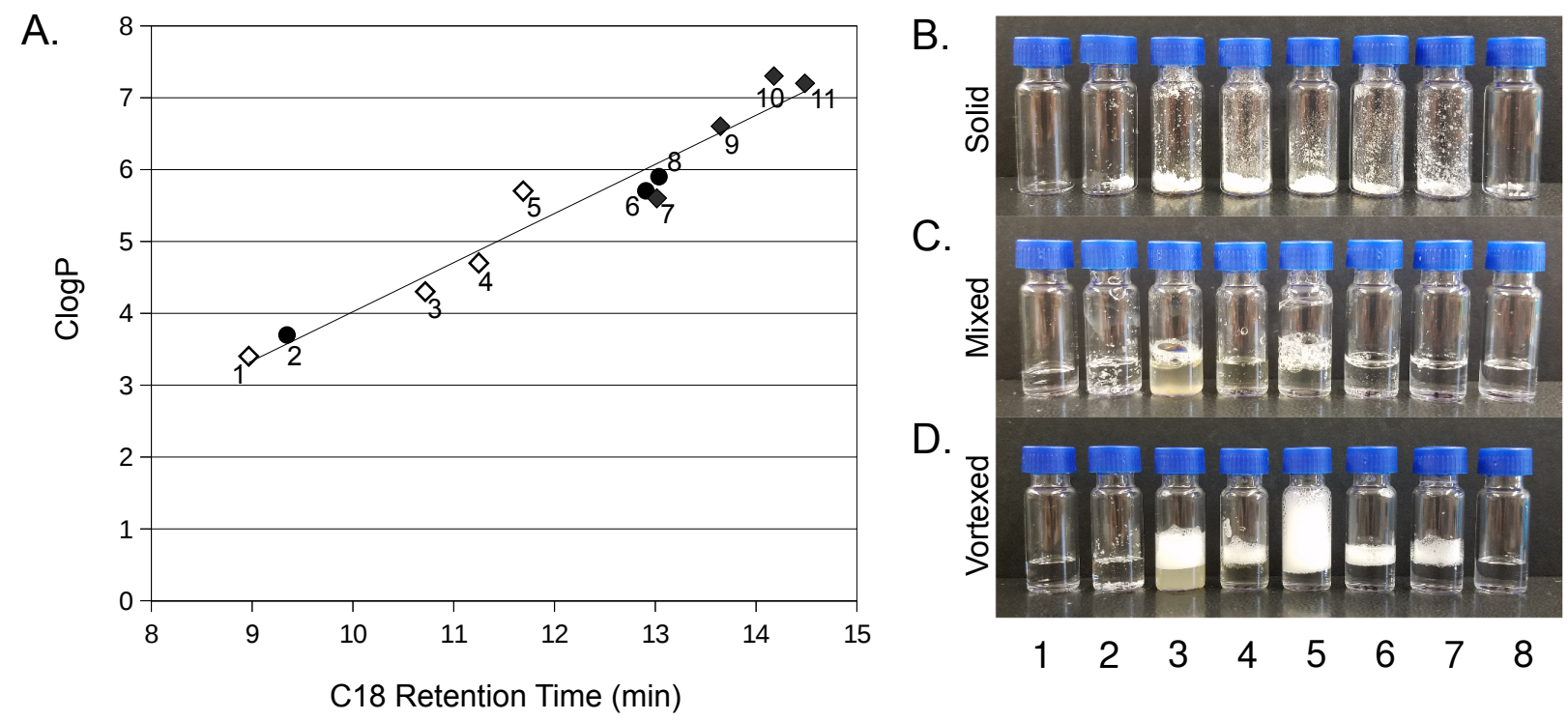
bioRxiv preprint doi: https://doi.org/10.1101/104349; this version posted January 30, 2017. The copyright holder for this preprint (which was not certified by peer review) is the author/funder, who has granted bioRxiv a license to display the preprint in perpetuity. It is made available under Hardman et al. 2017. Table 1aCC-BY-NC-ND 4.0 International license.

Table 1: Cannabinoid, cannaboside, and cannabinoid-metabolite ClogP values

\begin{tabular}{|c|c|}
\hline \# Compound & ClogP \\
\hline \begin{tabular}{l|l|}
1 & VB110 \\
\end{tabular} & 3.4 \\
\hline 2 11-COOH-Tetrahydrocannabinol Glucuronide & 3.7 \\
\hline \begin{tabular}{l|l}
3 & VB104
\end{tabular} & 4.3 \\
\hline \begin{tabular}{l|l}
4 & VB304
\end{tabular} & 4.7 \\
\hline \begin{tabular}{l|l}
5 & VB302
\end{tabular} & 5.7 \\
\hline \begin{tabular}{|l|l}
6 & $11-\mathrm{COOH}-T e t r a h y d r o c a n n a b i n o l$ \\
\end{tabular} & 5.7 \\
\hline \begin{tabular}{|l|l}
7 & Cannabidivarin \\
\end{tabular} & 5.6 \\
\hline \begin{tabular}{l|l|}
8 & $11-\mathrm{OH}-T e t r a h y d r o c a n n a b i n o l$ \\
\end{tabular} & 5.9 \\
\hline 9 Cannabidiol & 6.6 \\
\hline 10 Cannabinol & 7.3 \\
\hline 11 Tetrahydrocannabinol & 7.2 \\
\hline
\end{tabular}


bioRxiv preprint doi: https://doi.org/10.1101/104349; this version posted January 30, 2017. The copyright holder for this preprint (which was not certified by peer review) is the author/funder, who has granted bioRxiv a license to display the preprint in perpetuity. It is made available under aCC-BY-NC-ND 4.0 International license.

\section{Hardman et al. 2017: Supplemental Figure 1:}

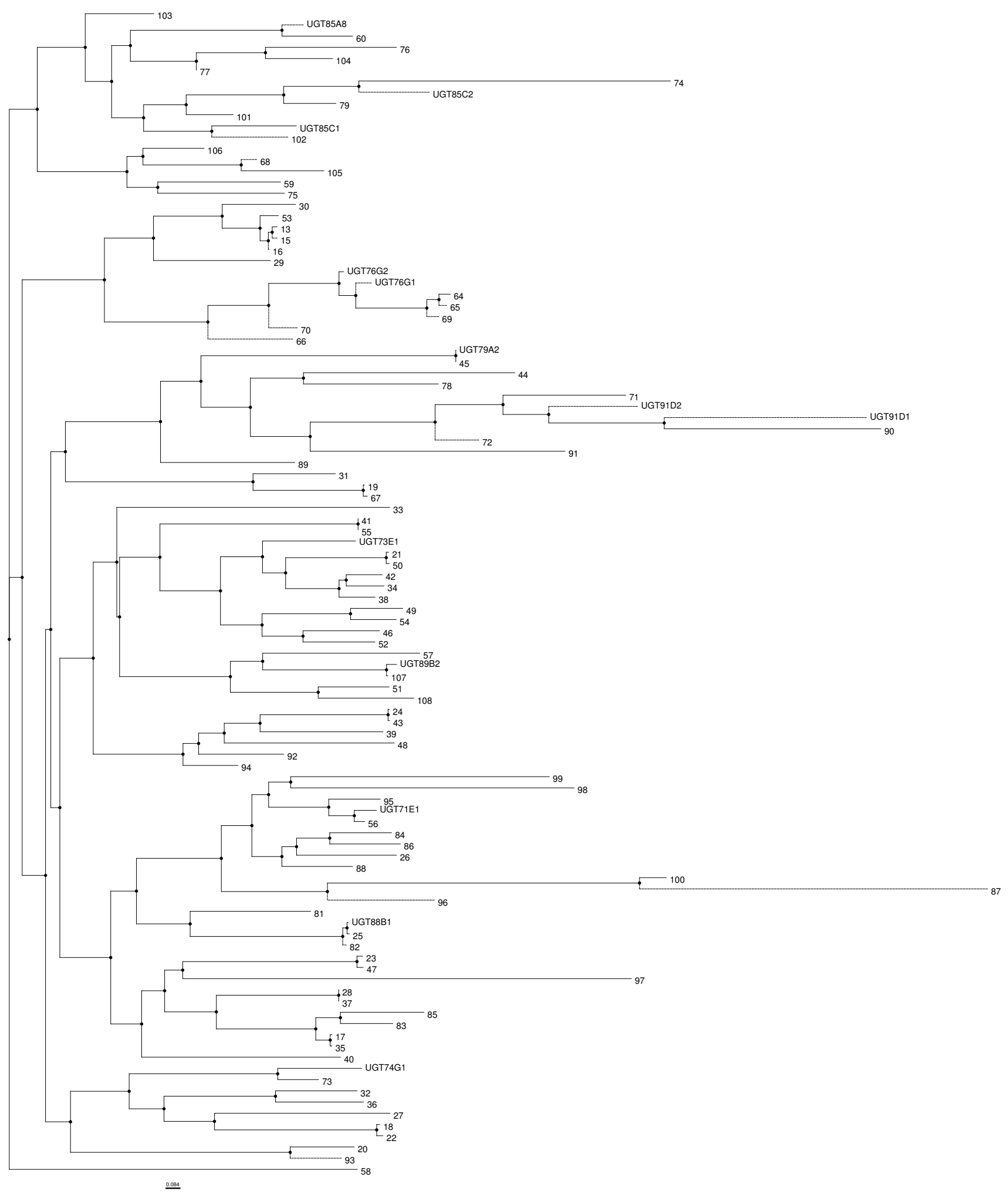




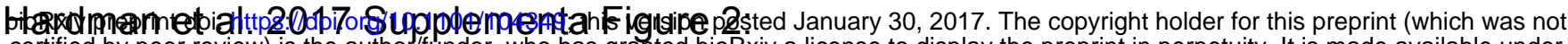
certified by peer review) is the author/funder, who has granted bioRxiv a license to display the preprint in perpetuity. It is made available under

A. \#:2 Ret.Time:Averaged 3.029-3.051(Scan\#:285-287)

BG Mode:Calc $2.912<->3.083(274<->290)$

Mass Peaks:313 Base Peak:315.30(46048) Polarity:Pos Segment1 - Event1

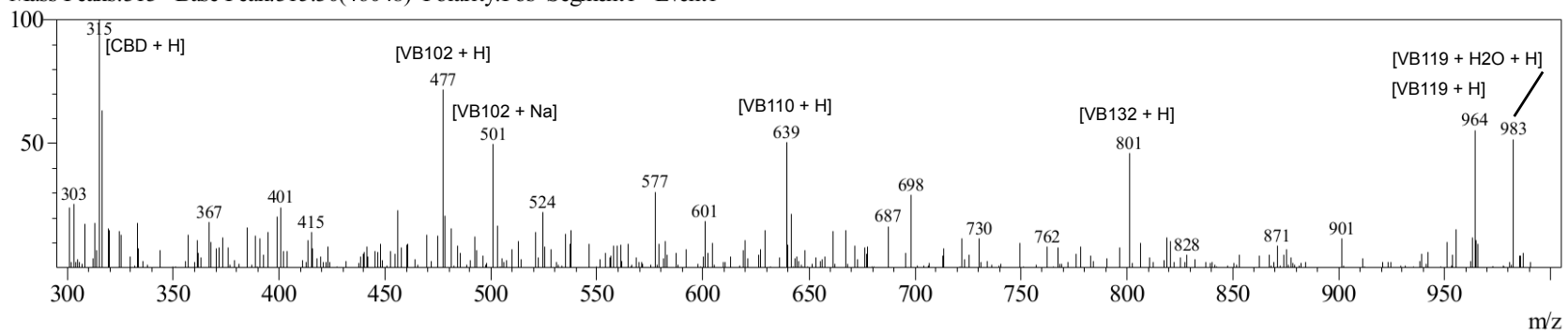

B.1 Ret.Time:Averaged 4.320-4.341(Scan\#:406-408)

BG Mode:Calc 4.117<->4.683(387<->440)

Mass Peaks:271 Base Peak:315.25(831547) Polarity:Pos Segment1 - Event1

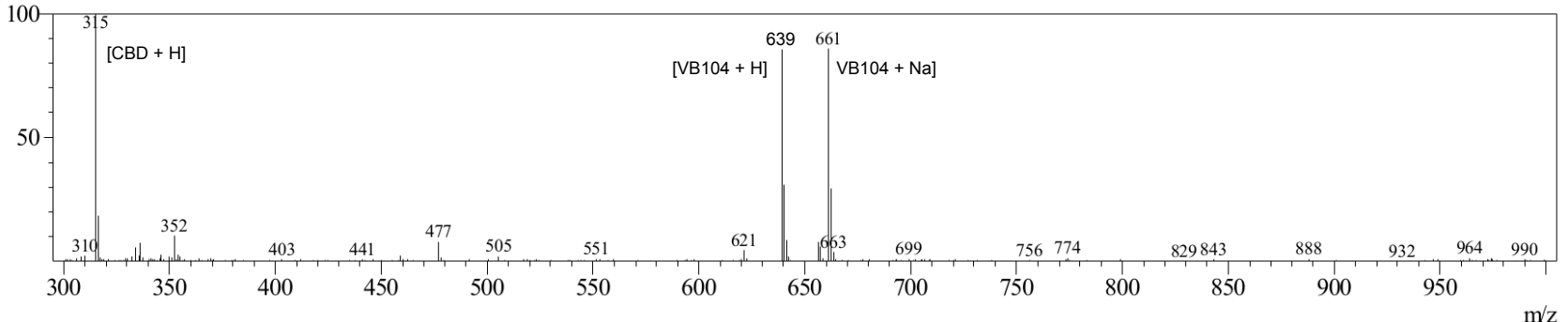

C. \#:1 Ret.Time:Averaged 4.309-4.331(Scan\#:405-407)

BG Mode:Calc 4.107<->4.704(386<->442)

Mass Peaks:304 Base Peak:315.25(854990) Polarity:Pos Segment1 - Event 1

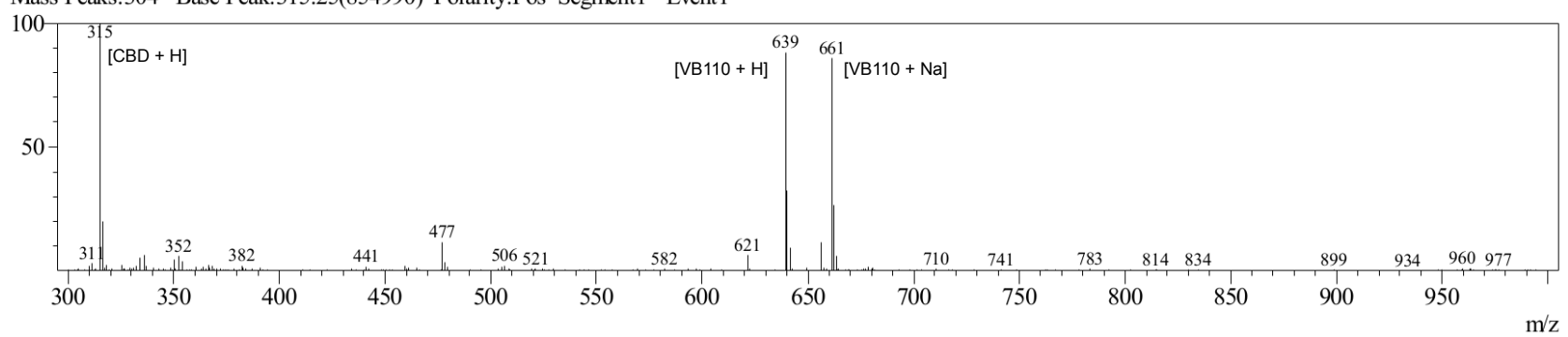

D. \#:1 Ret.Time:Averaged 4.309-4.331(Scan\#:405-407)

- BG Mode:Calc 4. 181<->4.565(393<->429)

Mass Peaks:261 Base Peak:639.30(100902) Polarity:Pos Segment1 - Event1

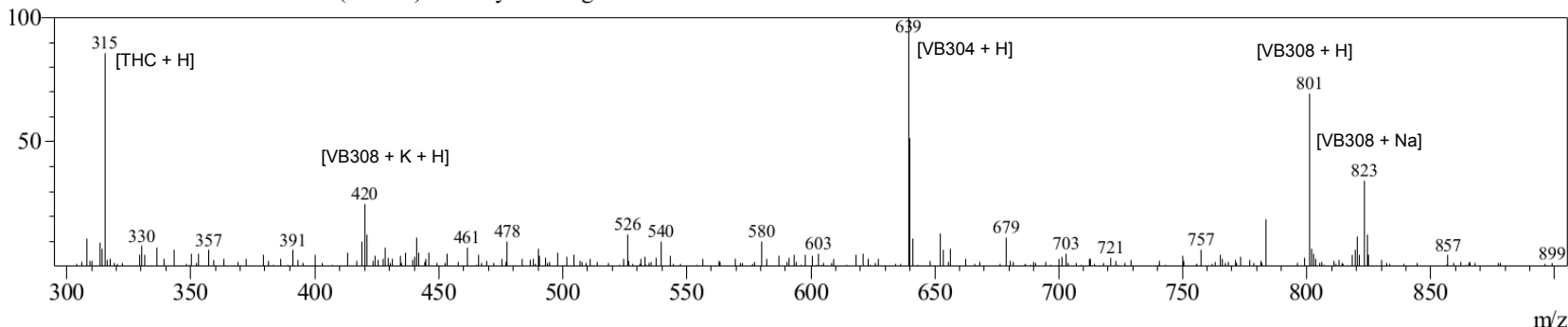


bioRxiv preprint doi: https://doi.org/10.1101/104349; this version posted January 30, 2017. The copyright holder for this preprint (which was not

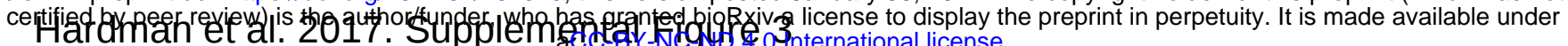

A.

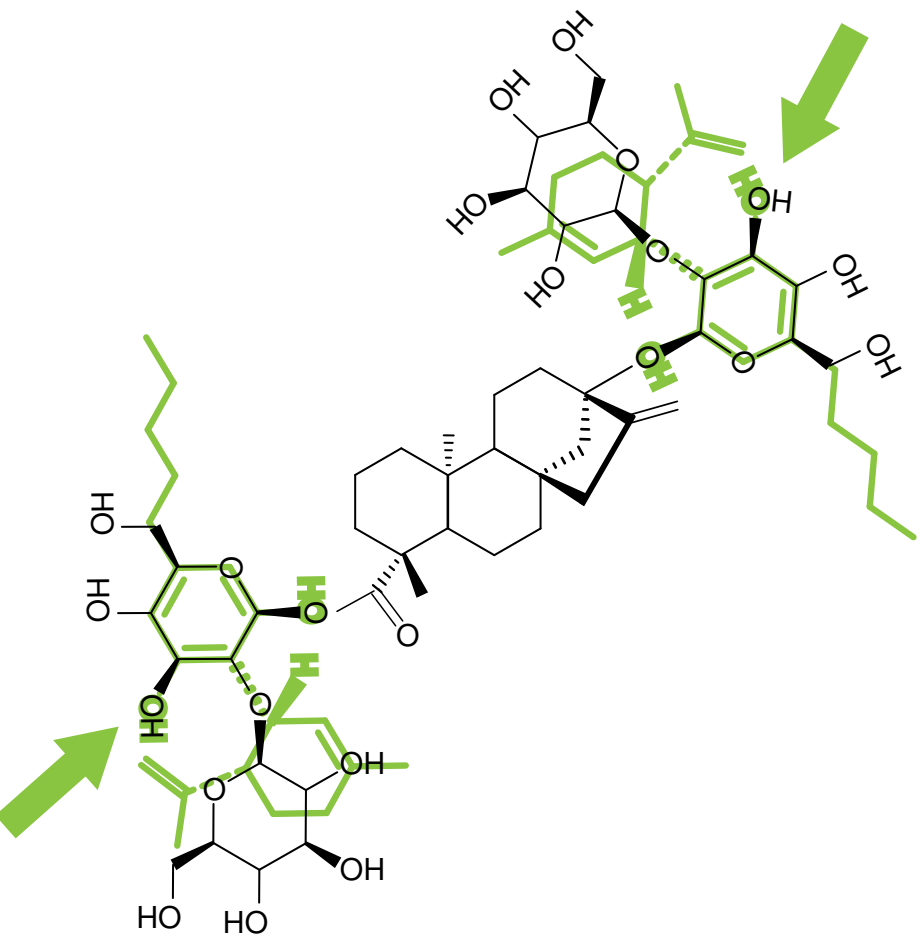

B.

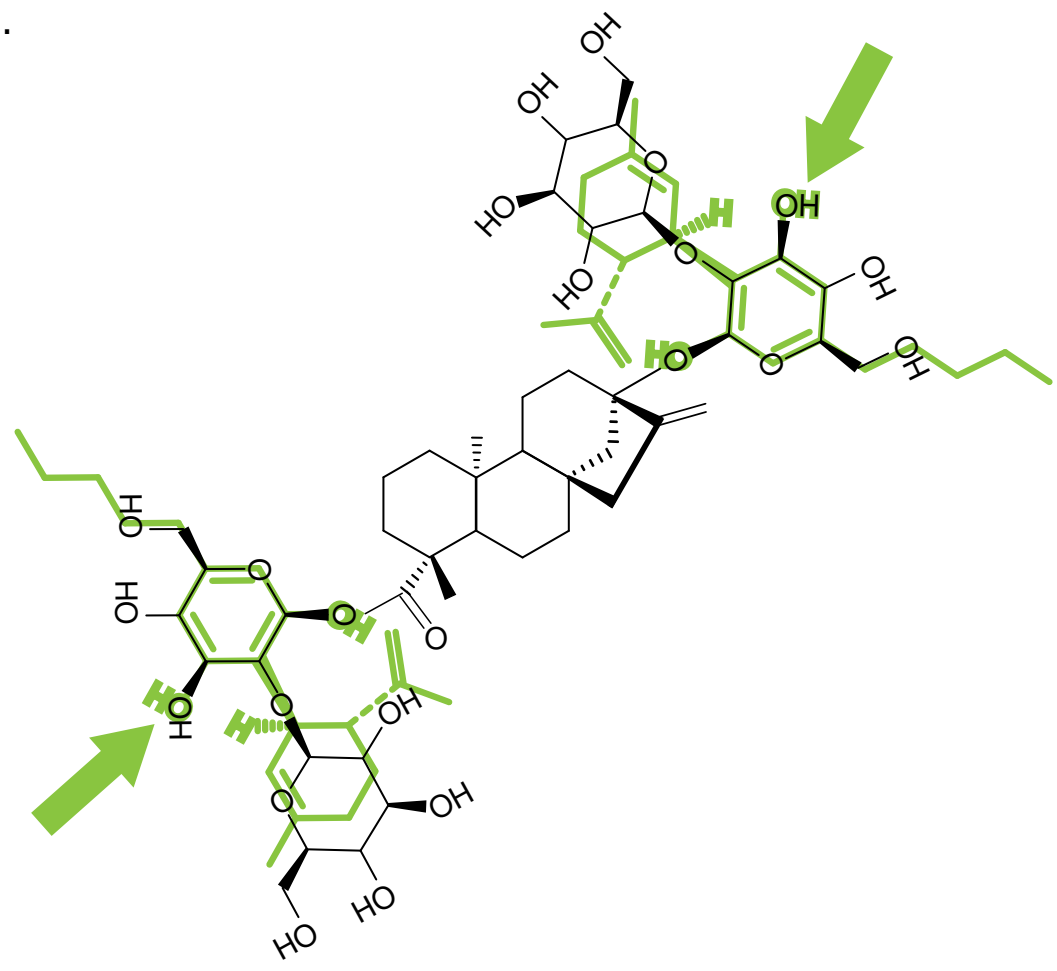



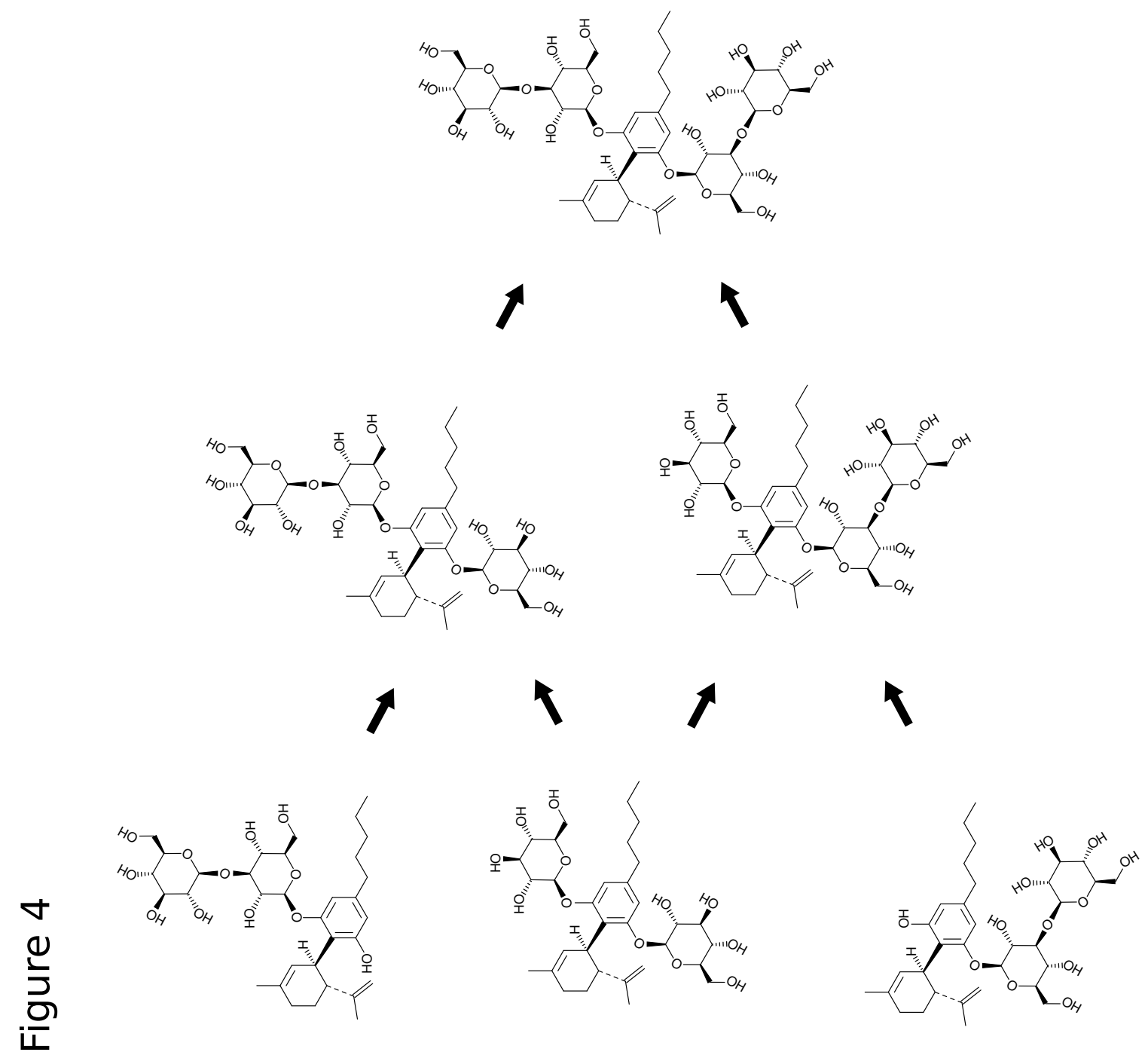

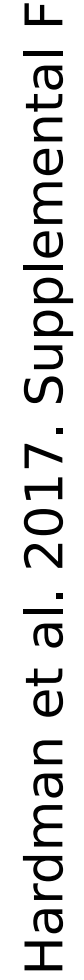
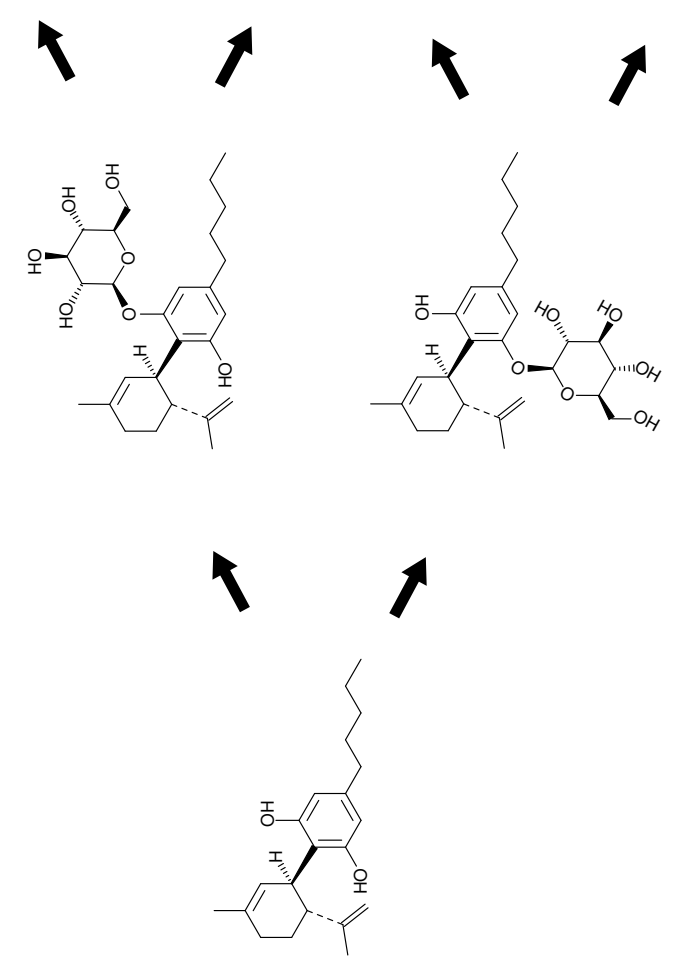
bioRxiv preprint doi: https://doi.org/10.1101/104349; this version posted January 30, 2017. The copyright holder for this preprint (which was not certified by peer review) is the author/funder, who has granted bioRxiv a license to display the preprint in perpetuity. It is made available under aCC-BY-NC-ND 4.0 International license.
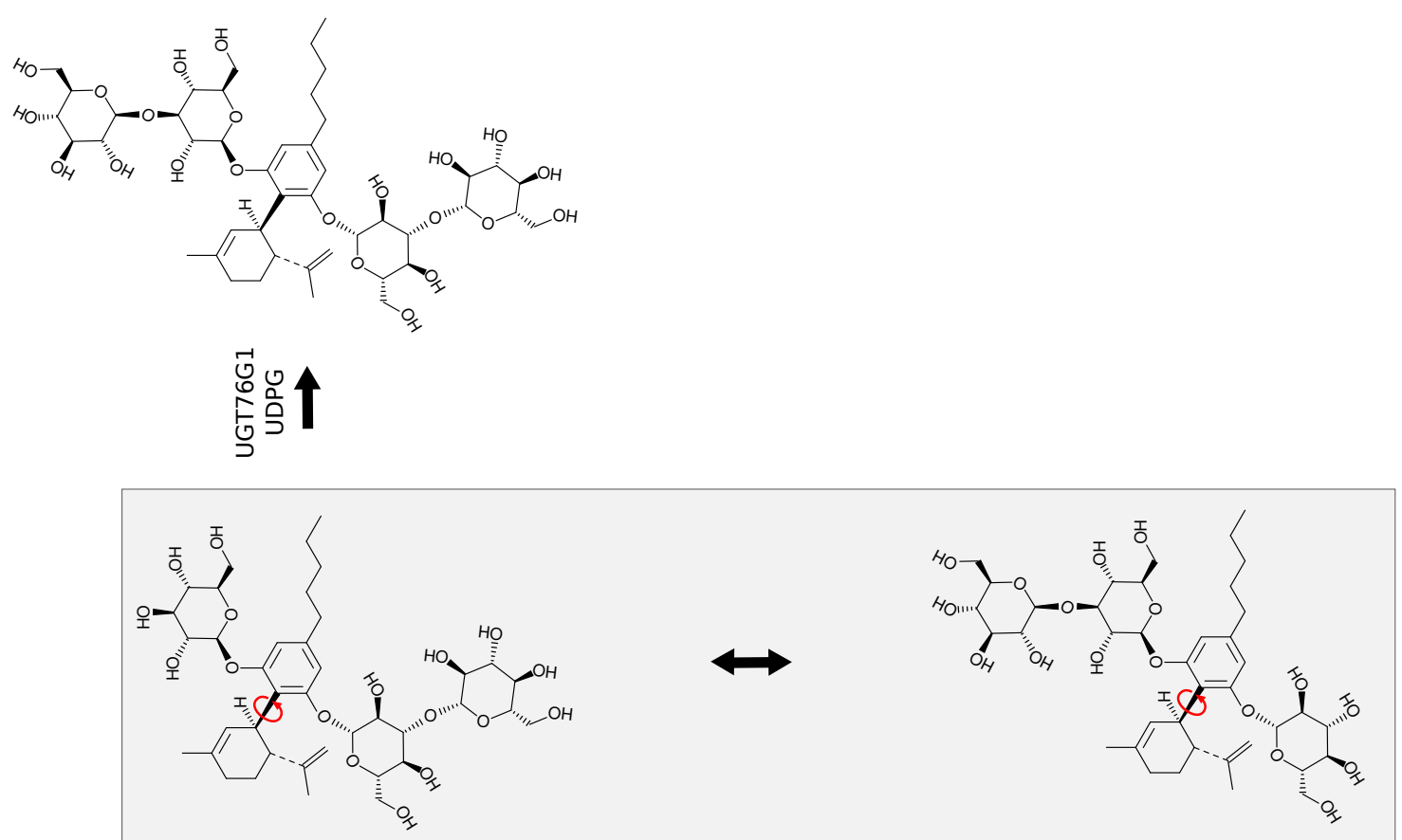

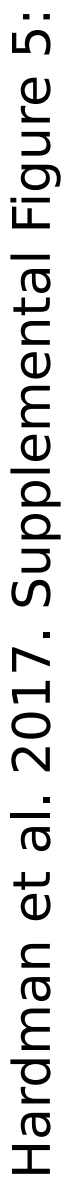

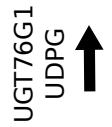

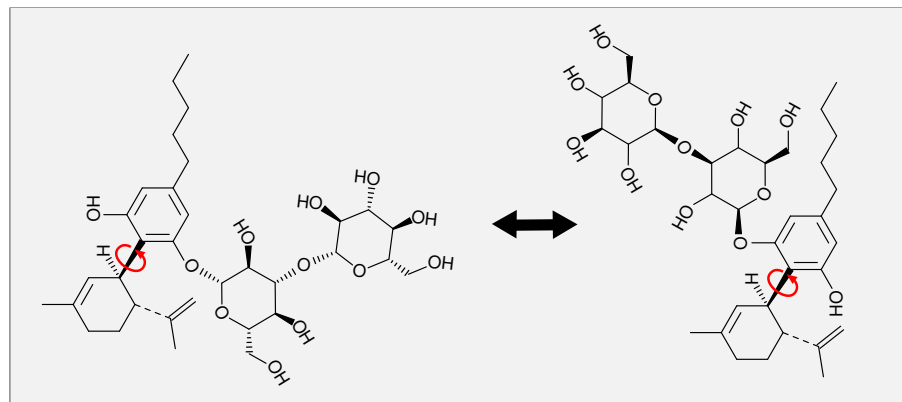

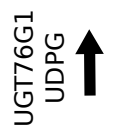

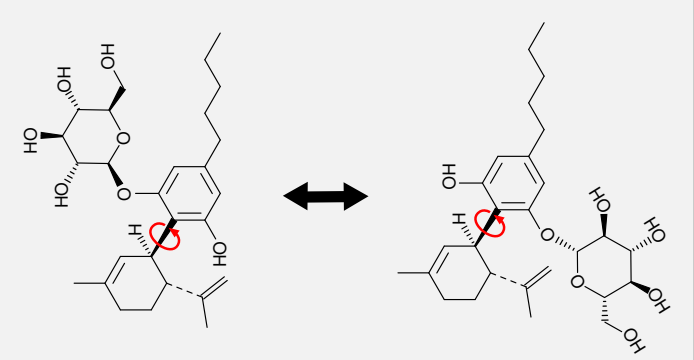

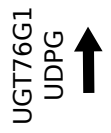

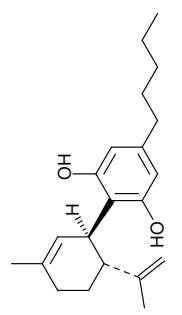




\section{Hardman et al. 2017. Supplemental Figure 6:}

Sequence 1: UGT76G1-5' primer with pLATE-11 LIC cloning sequence:

AGAAGGAGATATAACTATGGAAAATAAAACGGAGACCAC 3'

Sequence 2: UGT76G1-3' primer with GSG-HQHQHQ and pLATE-11 LIC cloning sequence:

GGAGATGGGAAGTCATTACTGGTGCTGGTGCTGGTGACCGCTACCCAACGATGAAATGT AAGAAA

Sequence 3: Os03g0702000-5' primer with GSG-HQHQHQ and pLATE-11 LIC cloning sequence:

AGAAGGAGATATAACTATGCATCAGCACCAACATCAGAGCGGTTCTATGGATAGTGGTTA CAGTTCTTCT

Sequence 4: Os03g070200-3' primer with pLATE-11 cloning sequence:

GGAGATGGGAAGTCATTATTAATCTTTGTACGAACGCAGTTG 